\title{
REPRESENTATION THEORY OF CENTRAL TOPOLOGICAL GROUPS
}

\author{
BY \\ SIEGFRIED GROSSER( $\left.{ }^{(}\right)$AND MARTIN MOSKOWITZ( $\left.{ }^{2}\right)$
}

By a central topological group we mean a group $G$ such that $G / Z$ is compact, where $Z$ (or $Z(G)$ ) denotes the center of $G$. A locally compact group satisfying this condition will be called a $[Z]$-group, and $[Z]$ denotes the class of these groups.

In [7] we developed the structure theory of [Z]-groups; in particular, we showed that every $[Z]$-group $G$ obeys the following Structure Theorem: $G=V \times H$ (direct product), where $V$ is a vector group and $H$ contains a compact open normal subgroup $K\left({ }^{3}\right)$.

Both the structure theory and, as will be shown in this paper, the representation theory of $[Z]$-groups generalize and unify in a natural manner the corresponding theories for compact groups on the one hand and for locally compact abelian groups on the other. In fact, many of the features common to these two classes appear in their natural setting only when viewed as being characteristic of $[Z]$-groups. In addition, there are strong indications that $[Z]$ marks the utmost degree of generality in which all these features are still present; not the least of these is the fact that the representation theory of $[Z]$-groups is essentially finite-dimensional. By contrast, that of the slightly larger class of $[F I A]^{-}$-groups is not; an $[F I A]^{-}$group being a locally compact group $G$ such that $\Im(G)$, the group of inner automorphisms, has compact closure in $\mathfrak{A}(G)$, the group of all topological group automorphisms of $G$, in the natural topology. The class $[F I A]^{-}$was introduced by R. Godement [4]. (For a full discussion of the relation between $[Z]$ and $[F I A]^{-}$, see [7].) The finite-dimensionality of representations referred to above in combination with the compactness condition which defines $[Z]$ allows us to obtain less general but considerably sharper results than those laid down by Godement in [6]. The present paper gives complete proofs of the results announced in Bull. Amer. Math. Soc. 72 (1966), 836-841, under the same title.

The paper is organized as follows. After establishing the basic definitions and a number of technical results in $\$ 1$ we proceed to the proof of the fundamental fact that continuous irreducible unitary Hilbert space representations of $[Z]$-groups are finite-dimensional (Theorem 2.1) and we obtain an orthogonality relation for the

Received by the editors December 21, 1965.

(1) Research partially supported by the National Science Foundation grant GP-3685.

(2) Research partially supported by the National Science Foundation, and Office of Army Research, Durham.

${ }^{(3)}$ This is Pontrjagin's classical structure theorem for locally compact abelian groups. 
coefficient functions of such representations. An application is made to a problem in the theory of discrete groups. In $\S 3$ we utilize the Gelfand-Raikov Theorem to prove that $[Z]$-groups are maximally almost periodic, i.e., belong to $[M A P]$; from this and results of [7] it follows that they satisfy the hypothesis of Takahashi's Duality Theorem [18]. Further results are that continuous functions and continuous central functions on a $[Z]$-group $G$ can be approximated uniformly on compact subsets of $G$ by representative functions and linear combinations of characters, respectively. In addition, the characters separate the conjugacy classes of $G$. $\$ 4$ contains a formula which characterizes, up to normalization, characters of irreducible representations of $[Z]$-groups. Then follow two irreducibility criteria for continuous finite-dimensional unitary representations (Theorem 4.3) and an application. We conclude this section with a result on nilpotent groups.

In $\$ 5$ we study the possibility of "extending" group characters (continuous homomorphisms into the circle group) from certain central subgroups of a group $G$ to continuous irreducible unitary representations of the whole group; $G$ is assumed to be either an $[M A P]$-group or a $[Z]$-group (Theorem 5.1, Corollary 1, and Theorem 5.5, respectively). In the latter case, essential use is made of the structure theorem for $[Z]$-groups as quoted above. This extension theorem utilizes and generalizes a result of Pontrjagin concerning the extension of group characters in abelian groups. Theorems 5.2 and 5.3 concern representations of bounded degree; they generalize results of I. Kaplansky [10]. Theorem 5.2 as well as the extension theorems depend on a result (Theorem 5.1) which analyses the irreducible representations of a compact subgroup of an [MAP]-group $G$ in terms of those of $G$. Next we characterize countable discrete $[Z]$-groups making use of a recent result of E. Thoma [19]. It should be remarked that the extension theorems derived here shed light on those found in $\S 3$ of [7] and yield an alternative proof of Theorem 3.1 independent of infinite-dimensional representation theory. $\$ 5$ closes with a theorem on locally faithful representations.

In $\S 6$ an orthogonality relation is derived which augments the one in $\S 2$. The final result is a criterion for equivalence of irreducible representations of a $[Z]$-group.

Finally the authors would like to thank $J$. Alperin for a number of useful discussions on representation theory of discrete groups.

1. Preliminaries. We begin with a section containing basic definitions and some results of a technical nature which we require for our investigation.

Definition. (1) Let $G$ be a topological group. Consider continuous finitedimensional irreducible unitary representations $\rho$ of $G$ on the complex vector space $V_{\rho}$; we denote the degree of $\rho$ by $d_{\rho}$ or $\operatorname{deg} \rho$ and the identity map on $V_{\rho}$ by $I_{d_{\rho}}$. Form equivalence classes of these representations, with respect to unitary equivalence, and choose one representation from each class. We denote by $\mathscr{R}$ (or $\mathscr{R}(G)$ ) the totality of all such representations. (2) If $\rho \in \mathscr{R}$ we denote by $\rho_{i j}$ the coordinate functions associated with $\rho$ relative to some orthonormal basis of $V_{\rho}$, by $\chi_{\rho}$ the 
character of $\rho$, and by $\mathfrak{X}$ (or $\mathfrak{X}(G)$ ) the family of all such characters. (3) We denote by $\mathscr{F}_{c}\left(\right.$ or $\mathscr{F}_{c}(G)$ ), $\mathscr{F}_{u}$ (or $\mathscr{F}_{u}(G)$ ), and $\mathscr{F}_{c_{0}}\left(\right.$ or $\mathscr{F}_{c_{0}}(G)$ ), respectively, the algebras of complex-valued functions on $G$ which are continuous, (left) uniformly continuous $\left({ }^{4}\right)$, and continuous with compact support; by $\mathscr{F}_{r}\left(\right.$ or $\mathscr{F}_{r}(G)$ ) the subalgebra of $\mathscr{F}_{u}$ consisting of representative functions associated with representations in $\mathscr{R}$, and by $\mathscr{F}_{z}\left(\right.$ or $\mathscr{F}_{z}(G)$ ) the subalgebra of $\mathscr{F}_{c}$ consisting of the central functions-a central function being one which is constant on the conjugacy classes of $G$. (4) As usual, a subset $S$ of $G$ is called invariant if it is stable under the inner automorphisms of $G$. In general, we denote by $G \square S$ the orbit of $S$ under the inner automorphisms of $G$. If $f \in \mathscr{F}_{c}$ and $x \in G$ then $f^{x}$ denotes the left translate of $f$ by $x$, i.e., $f^{x}(y)=f(x y)$; and $x \Delta f$ denotes the conjugate of $f$ by $x$, i.e., $(x \Delta f)(y)$ $=f\left(x y x^{-1}\right)$. The restriction of $f$ to a subset $S$ of $G$ is denoted by $f_{S}$. If $S$ is a subset of $G$ on which $f$ is bounded, $\|f\|_{S}$ stands for lub $\{|f(x)| / x \in S\}$. The support of $f$ is denoted by $\operatorname{Supp} f$. Finally, $\int_{G / Z} d \dot{x}$ denotes the normalized Haar integral on $G / Z$, and $\int_{G} d x$ and $\int_{Z} d z$ are left invariant Haar integrals on $G$ and $Z$ respectively; normalized so that $\int_{G}=\int_{Z} \int_{G / Z}$; the associated Haar measures are denoted by $\mu_{G / Z}, \mu_{G}$, and $\mu_{\mathrm{Z}}$.

All notation not explicitly defined will be standard. At times elements of $\mathscr{F}_{c}(G / Z)$ will be regarded as elements of $\mathscr{F}_{c}(G)$.

THEOREM 1.1. Assume that $G \in[Z]$. There exists a linear operator \#:

with the following properties:

$$
\mathscr{F}_{u} \rightarrow \mathscr{F}_{u} \cap \mathscr{F}_{z}
$$

(1) If $f \in \mathscr{F}_{u} \cap \mathscr{F}_{z}$ then $f^{\#}=f$.

(2) If $f \in \mathscr{F}_{c_{0}}$ then $\operatorname{Supp} f^{\#} \subseteq G \square \operatorname{Supp} f$, and $f^{\#} \in \mathscr{F}_{c_{0}}$.

(3) If $F$ is a compact invariant subset of $G$ then $\left\|f^{\#}\right\|_{F} \leqq\|f\|_{F}$.

(4) If $f \in \mathscr{F}_{r}$ then $f^{\#} \in \mathscr{F}_{r}$.

Proof. For $f$ in $\mathscr{F}_{u}$ and $y$ in $G$, define

$$
f^{\#}(y)=\int_{G / Z}(\dot{x} \Delta f)(y) d \dot{x}\left({ }^{5}\right) .
$$

Evidently, for each $y,(x \Delta f)(y)$ is a continuous function on $G / Z$. One checks easily that \# is linear. Now we have

$$
f^{\#}\left(t y t^{-1}\right)=\int_{G / Z}(\dot{x} \Delta f)\left(t y t^{-1}\right) d \dot{x}=\int_{G / Z}((x t) \cdot \Delta f)(y) d \dot{x}=\int_{G / Z}(\dot{x} \dot{x} \Delta f)(y) d \dot{x},
$$

(4) Since, as was shown in [7], $G$ has small invariant neighborhoods of 1, i.e., $G \in[S I N]$, as is well known, the left and right uniform structures on $G$ coincide.

(5) It follows from Theorem 1.1 that in the case of compact groups the \# operator as defined above; coincides with an operator used implicitly by Pontrjagin in [16]. (In this context, see the Remark following Theorem 1.2.) Segal [17] explicitly defines an operation like \# when considering direct sums of compact and abelian groups while in work of Godement [4], [6] the \# operator is defined in a slightly more general setting. 
and by the translation invariance of $\int_{G / Z} d \dot{x}$ the last expression equals $f^{\#}(y)$. Hence $f^{\#} \in \mathscr{F}_{c}(G)$. Let $\varepsilon>0$ be given and choose a symmetric neighborhood $U_{\varepsilon}$ of 1 in $G$ with the property that if $y_{2}^{-1} y_{1} \in U_{\varepsilon}$ then $\left|f\left(y_{1}\right)-f\left(y_{2}\right)\right|<\varepsilon$. Since $G$ has small invariant neighborhoods of 1 (by Theorem 4.2 of [7]), we may assume that $U_{\varepsilon}$ is invariant. Then

$$
\left|f^{\#}\left(y_{1}\right)-f^{\#}\left(y_{2}\right)\right| \leqq \int_{G / Z}\left|(\dot{x} \Delta f)\left(y_{1}\right)-(\dot{x} \Delta f)\left(y_{2}\right)\right| d \dot{x} .
$$

But if $y_{2}^{-1} y_{1} \in U_{\varepsilon}$ then one sees easily from the above that

$$
\left|(\dot{x} \Delta f)\left(y_{1}\right)-(\dot{x} \Delta f)\left(y_{2}\right)\right|<\varepsilon,
$$

for all $\dot{x}$ in $G / Z$. Hence it follows that $f^{\#}$ is uniformly continuous. Thus

$$
f^{\#} \in \mathscr{F}_{u} \cap \mathscr{F}_{z} \text {. }
$$

(1) If $f \in \mathscr{F}_{u} \cap \mathscr{F}_{z}$ then it follows immediately from the definition of \# that $f^{\#}(y)=f(y)$.

(2) If, for a fixed $y,(i \Delta f)(y)=0$, for all $i$ in $G / Z$, then $f^{\#}(y)=0$. Hence if $f^{\#}(y) \neq 0$, there is a $t$ in $G$ such that $f\left(t y t^{-1}\right) \neq 0$. Let $x=t y t^{-1}$. Then $y=t^{-1} x t$ and $f(x) \neq 0$. Thus

$$
\left\{y / f^{\#}(y) \neq 0\right\} \subseteq G \mathbf{m}\{x / f(x) \neq 0\} \subseteq G \mathbf{m} \operatorname{Supp} f .
$$

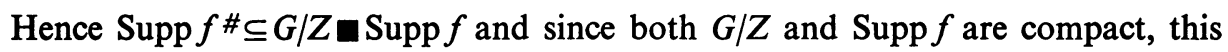
implies that $\operatorname{Supp} f^{\#}$ is compact.

(3) We have

$$
\operatorname{lub}\left\{\left|f^{\#}(y)\right| / y \in F\right\} \leqq \operatorname{lub}\left\{\int_{G / Z}|(\dot{x} \Delta f)(y)| d \dot{x} / y \in F\right\} .
$$

The integrand is $\leqq\|f\|_{G \backsim F}$. Since $F$ is invariant, this implies that $\|f \#\|_{F} \leqq\|f\|_{F}$.

(4) Since \# is linear, it suffices to show that $\rho_{i j}^{\#} \in \mathscr{F}_{r}$, where $\rho \in \mathscr{R}$ and $\rho(x)=\left(\rho_{i j}(x)\right)$. Since $\rho$ is a representation,

$$
\left(\dot{x} \Delta \rho_{i j}\right)(y)=\sum_{k, l=1}^{d_{\rho}} \rho_{i k}(x) \rho_{k l}(y) \rho_{l j}\left(x^{-1}\right),
$$

where $x$ is an element of $G$ belonging to the inverse image of $\dot{x}$. Now $\rho_{i k}(x) \rho_{l j}\left(x^{-1}\right)$ is a function on $G / Z$. For if $x \in G$ and $z \in Z$, then

$$
\begin{aligned}
\rho_{i k}(x z) \rho_{l j}\left((x z)^{-1}\right) & =\left(\rho(x z) \otimes \rho\left(z^{-1} x^{-1}\right)\right)_{i k l j} \\
& =\left(\left(\rho(x) \otimes \rho\left(x^{-1}\right)\right)\left(\rho(z) \otimes \rho\left(z^{-1}\right)\right)\right)_{i k l j} .
\end{aligned}
$$

But $\rho(x) \rho(z)=\rho(z) \rho(x)$, and since $\rho$ is irreducible it follows from Schur's Lemma that $\rho(z)=\lambda(z) I_{d_{\rho}}$, for each $z$ in $Z$, so that $\rho(z) \otimes \rho\left(z^{-1}\right)=I_{d_{\rho}^{2}}$. Thus

$$
\rho_{i k}(z x) \rho_{l j}\left(z^{-1} x^{-1}\right)=\left(\rho(x) \otimes \rho\left(x^{-1}\right)\right)_{i k l j}=\rho_{i k}(x) \rho_{l j}\left(x^{-1}\right) .
$$

Hence

$$
\rho_{i k}(x) \rho_{l j}\left(x^{-1}\right) \rho_{k l}(y)=g_{i k l j}(\dot{x}) \rho_{k l}(y)
$$


and therefore

$$
\rho_{i j}^{\#}(y)=\sum_{k, l=1}^{d \rho} \rho_{k l}(y)\left(\int_{G / Z} g_{i k l j}(\dot{x}) d \dot{x}\right),
$$

which proves (4).

We require the following

LEMMA. In a $[Z]$-group $G$ every compact set is contained in a compact invariant neighborhood of 1 . Moreover, $G=U Z$, where $U$ is a compact invariant symmetric neighborhood of 1 .

Proof. If $C$ is a compact subset of $G$ then $C$ is contained in a compact neighborhood $E$ of 1 . Since $G / Z$ is compact, the set $G \square E$ is also compact and is an invariant neighborhood of 1 containing $C$.

Since $G / Z$ is compact and $G$ is locally compact, $G=C Z$, where $C$ is a compact subset of $G$. Then $C$ is contained in a compact invariant symmetric neighborhood $U$ of 1 , and $G=U Z$.

THEOREM 1.2( $\left.{ }^{6}\right)$. If $G$ is a [Z]-group then there is a nonnegative function $w \in \mathscr{F}_{c_{0}} \cap \mathscr{F}_{z}$ with the property that, for any function $f$ in $\mathscr{F}_{c}(G / Z)$,

$$
\int_{G / Z} f(\dot{x}) d \dot{x}=\int_{G} w(x) f(x) d x .
$$

We shall refer to such a function as a weighting function.

Proof. Write $G=U Z$ as in the Lemma. Since $U$ is compact, there is a nonnegative function $g$ in $\mathscr{F}_{c_{0}}(G)$ satisfying $g(u)=1$, for all $u$ in $U$. Since $g \in \mathscr{F}_{u}(G)$, we may apply \# to $g$. Because of the invariance of $U$ it follows from the definition of \# that

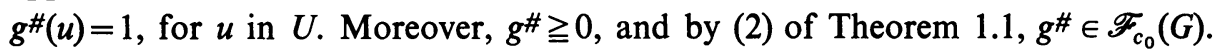
Hence, for the purpose of proving the present theorem, we may assume that $g \in \mathscr{F}_{z}(G)$.

For each $x$ in $G$, consider $\left(g^{x}\right)_{z}$. Evidently, this function is nonnegative and has compact support in $Z$. Define $h(x)=\int_{Z}\left(g^{x}\right)_{z}(z) d z$. Since $\left(g^{x}\right)_{z} \in \mathscr{F}_{u}(Z), h \in \mathscr{F}_{c}(G)$. Let $x \in G$ and write $x=u z$, where $u \in U$ and $z \in Z$. Then $\left(g^{x}\right)_{z}\left(z^{-1}\right)=g\left(x z^{-1}\right)$ $=g(u)=1$, so that $\left(g^{x}\right)_{z}$ is positive at $z^{-1}$; hence $h(x)>0$, for every $x$ in $G$. Define $w(x)=g(x) / h(x)$. Then $w \in \mathscr{F}_{c_{0}}$ and $w$ is nonnegative. For $z_{1}$ in $Z$, we have

$$
h^{x}\left(z_{1}\right)=h\left(x z_{1}\right)=\int_{z} g^{x z_{1}}(z) d z=\int_{z} g^{x}(z) d z=h(x),
$$

$\left.{ }^{(}\right)$The technique of proof employed here is similar to that of P. Cartier in [1]. We remark that his proof applies to a more general situation than the one considered here but cannot yield the fact that the weighting function is central. In any case, such functions have been used in classical Fourier analysis for half a century in converting integrals over a period of a periodic function to integrals over the whole real line. 
so that $\left(h^{x}\right)_{z}$ is the constant function with value $h(x)$. Therefore,

$$
\int_{z}\left(w^{x}\right)_{z}(z) d z=\int_{z}\left(\left(g^{x}\right)_{z} /\left(h^{x}\right)_{z}\right)(z) d z=(1 / h(x)) \int_{z}\left(g^{x}\right)_{z}(z) d z=1,
$$

for each $x$ in G. Moreover,

$$
g^{x y x^{-1}}(z)=g\left(x y x^{-1} z\right)=g\left(x y z x^{-1}\right)=g(y z)=g^{y}(z),
$$

since $g$ is central. Thus $\left(g^{x y x^{-1}}\right)_{z}=\left(g^{y}\right)_{z}$ and therefore $h \in \mathscr{F}_{z}(G)$, by the definition of $h$. It follows that $w \in \mathscr{F}_{z}(G)$.

Now assume $f \in \mathscr{F}_{c}(G / Z)$ and let $x \in G$. Clearly $\left((w f)^{x}\right)_{z}=\left(w^{x}\right)_{Z}\left(f^{x}\right)_{z}$ and hence $\int_{Z}\left((w f)^{x}\right)_{Z}(z) d z=\int_{Z}\left(w^{x}\right)_{z}(z)\left(f^{x}\right)_{z}(z) d z$.

Now, for $z$ in $Z, f^{x}(z)=f(x z)=f(x)$, so that $\left(f^{x}\right)_{z}$ is the constant function on $Z$ with value $f(x)$. From this and from the above one sees immediately that $\int_{z}\left((w f)^{x}\right)_{z}(z) d z=f(x)$, for every $x$ in $G$. But then

$$
\int_{G}(w f)(x) d x=\int_{G / Z}\left(\int_{Z}\left((w f)^{x}\right)_{z}(z) d z\right) d \dot{x}=\int_{G / Z} f(\dot{x}) d \dot{x} .
$$

REMARK. We note that by means of this weighting function one can convert all relations involving integrals over $G / Z$ into relations involving integrals over $G$. For example, the \# operator is given by

$$
f^{\#}(y)=\int_{G} w(x) f\left(x y x^{-1}\right) d x .
$$

Furthermore, the proof of Theorem 1.2 shows that if $G$ is itself compact then $w$ can be chosen to be constant. Since the normalization of the Haar integral on $G / Z$ forces that constant to be 1 , it follows that if $G$ is compact and $f \in \mathscr{F}_{c}(G / Z)$ then $\int_{G} f(x) d x=\int_{G \mid Z} f(\dot{x}) d \dot{x}$. In particular, the \# operator, in this case, is given by

$$
f^{\#}(y)=\int_{G} f\left(x y x^{-1}\right) d x .
$$

Proposition. If $G$ has a compact invariant neighborhood of 1 then $G$ is unimodular. In particular, $[Z]$-groups are unimodular $\left({ }^{7}\right)$.

The (easy) proof of this proposition will be omitted.

Definition. Let $G$ be a locally compact group. We say that $G$ has small central functions if for each neighborhood $U$ of 1 in $G$ there is a nonnegative function $f_{U} \in \mathscr{F}_{c_{0}} \cap \mathscr{F}_{z}$ satisfying the conditions

(1) $\operatorname{Supp} f_{U} \subseteq U$,

(2) $f_{U}(1)>0$.

THEOREM 1.3. $G \in[S I N]$ if and only if $G$ has small central functions. In particular, if $G \in[Z]$, then $G$ has this property.

(7) This is clear from $\left(^{4}\right)$. 
Proof. Assume $G \in[S I N]$ and let $U$ be a compact neighborhood of 1 . Let $S$ be a compact invariant symmetric neighborhood of 1 in $G$ that satisfies $S^{2} \subseteq U$. Define $f_{U}: G \rightarrow \mathbf{R}$ by

$$
f_{U}(x)=\mu_{G}(S \cap x S) \quad(x \in G) .
$$

Clearly, $\infty>f_{U}(1) \geqq f_{U}(x) \geqq 0$. Since $x \notin S S^{-1}$ implies $S \cap x S=\varnothing$, it follows that Supp $f_{U} \subseteq U$. Using the invariance of $S$ and the unimodularity of $G$, one shows by a direct calculation that $f_{U}(x)=f_{U}\left(y x y^{-1}\right)$, for all $x, y$ in $G$, i.e., that $f_{U}$ is central.

If $X$ is a subset of $G$ denote by $\phi_{X}$ its characteristic function. Then $\phi_{S}\left(\phi_{S}\right)^{x^{-1}}$ is the characteristic function of $S \cap x S$, so that $f_{U}(x)=\int_{G} \phi_{S}(y)\left(\phi_{S}\right)^{x^{-1}}(y) d y$. Since $S$ is symmetric, it follows that $\phi_{S}\left(x^{-1} y\right)=\phi_{S}\left(y^{-1} x\right)$, so that

$$
f_{U}(x)=\int_{G} \phi_{S}(y) \phi_{S}\left(y^{-1} x\right) d y=\phi_{S} * \phi_{S}(x)
$$

where $*$ denotes the convolution. Since the convolution of a bounded function with an $L_{1}$-function is uniformly continuous [11], $f_{U}$ is continuous.

Conversely, assume that $G$ has small central functions. Let $U$ be a neighborhood of 1 and $f_{U}$ the corresponding function. Since $f_{U}$ is continuous, there exists a neighborhood $S$ of 1 such that, for all $s$ in $S$, (1/2) $f_{U}(1) \leqq f_{U}(s) \leqq(3 / 2) f_{U}(1)$. Then $f_{U}(s)>0$, so that $S \subseteq U$. Since $f_{U}$ is central, we have $f_{U}\left(x s x^{-1}\right)=f_{U}(x)>0$, for every $x$ in $G$. Hence $x S x^{-1} \subseteq U$, i.e., $S \subseteq x^{-1} U x$, and therefore $S \subseteq \bigcap_{x \in G} x^{-1} U x$. Then $\bigcap_{x \in G} x^{-1} U x$ is an invariant neighborhood of 1 contained in $U$.

2. Finite-dimensionality of representations and an orthogonality relation. By modifying a computation of Nachbin's [14] which yields the finite-dimensionality of irreducible unitary representations of compact groups we get a generalization of this fact for $[Z]$-groups as well as an orthogonality relation for coefficients of an irreducible unitary representation which likewise generalizes the corresponding one for compact groups.

Proposition. Let $G \in[Z]$ and let $\rho$ be a continuous irreducible unitary representation of $G$ on the complex Hilbert space $V$. Then:

(1) For each choice of $u, v, u^{\prime}, v^{\prime}$ in $V$ the function defined by $\left\langle\rho_{x}(u), v\right\rangle\left\langle\rho_{x}\left(u^{\prime}\right), v^{\prime}\right\rangle^{-}$ (where $x \in G$ and - denotes complex conjugation) is in $\mathscr{F}_{c}(G / Z)$.

(2) To $\rho$ corresponds a positive real number $c_{\rho}=\int_{G / Z}\left|\left\langle\rho_{x}(u), v\right\rangle\right|^{2} d \dot{x}$, where $u$ and $v$ are any vectors in $V$ of norm 1.

$$
\int_{G / Z}\left\langle\rho_{x}(u), v\right\rangle\left\langle\rho_{x}\left(u^{\prime}\right), v^{\prime}\right\rangle^{-} d \dot{x}=c_{\rho}\left\langle u, u^{\prime}\right\rangle\left\langle v, v^{\prime}\right\rangle^{-},
$$

for all $u, v, u^{\prime}, v^{\prime}$ in $V$.

Proof. (1) Let $z \in Z(G)$. Since $\rho$ is a representation, $\rho(x) \rho(z)=\rho(z) \rho(x)$, for each $x$ in $G$. Hence, by the Hilbert space version of Schur's Lemma $[11,15], \rho(z)=\lambda(z) I$, 
where $\lambda(z) \in \mathbf{C}$ and $I$ is the identity map of $V$. Since $\rho(z)^{-1}=\rho(z)^{*}$, we have $|\lambda(z)|^{2}=1$. Thus

$$
\begin{aligned}
\left.\left\langle\rho_{z} \rho_{x}(u), v\right\rangle\left\langle\rho_{z} \rho_{x}\left(u^{\prime}\right), v^{\prime}\right)\right\rangle^{-} & =\lambda(z)\left\langle\rho_{x}(u), v\right\rangle[\lambda(z)]^{-}\left\langle\rho_{x}\left(u^{\prime}\right), v^{\prime}\right\rangle^{-} \\
& =\left\langle\rho_{x}(u), v\right\rangle\left\langle\rho_{x}\left(u^{\prime}\right), v^{\prime}\right\rangle^{-} .
\end{aligned}
$$

Since $\rho$ is weakly continuous, the function in question is in $\mathscr{F}_{c}(G / Z)$.

Now we shall establish (2) and (3). Consider

$$
\phi\left(u, v, u^{\prime}, v^{\prime}\right)=\int_{G / Z}\left\langle\rho_{x}(u), v\right\rangle\left\langle\rho_{x}\left(u^{\prime}\right), v^{\prime}\right\rangle-d \dot{x} .
$$

For fixed $u, u^{\prime}$, this function is linear in $v^{\prime}$ and conjugate linear in $v$. Moreover because of the Schwarz Inequality and the fact that $\rho$ is unitary, $\left|\phi\left(u, v, u^{\prime}, v^{\prime}\right)\right| \leqq$ $\|u\|\|v\|\left\|u^{\prime}\right\|\left\|v^{\prime}\right\|$. Consequently, for fixed $u, u^{\prime}, \phi\left(u, v, u^{\prime}, v^{\prime}\right)$ can be represented by a bounded operator. Specifically, there exists a family $B_{u, u^{\prime}}$ of bounded operators on $V$ satisfying $\left\langle B_{u, u^{\prime}}\left(v^{\prime}\right), v\right\rangle=\phi\left(u, v, u^{\prime}, v^{\prime}\right)$. But then, because of the invariance of the Haar integral on $G \mid Z$, we have $\left\langle B_{u, u^{\prime}}\left(\rho_{y}\left(v^{\prime}\right)\right), v\right\rangle=\int_{G / Z}\left\langle\rho_{y x}(u), v\right\rangle\left\langle\rho_{y x}\left(u^{\prime}\right), \rho_{y}\left(v^{\prime}\right)\right\rangle^{-} d \dot{x}$. However, since $\left\langle\rho_{y x}\left(u^{\prime}\right), \rho_{y}\left(v^{\prime}\right)\right\rangle=\left\langle\rho_{x}\left(u^{\prime}\right), v^{\prime}\right\rangle$ and $\left\langle\rho_{y x}(u), v\right\rangle=\left\langle\rho_{x}(u), \rho_{y}^{*}(v)\right\rangle$, it follows that

$$
\left\langle B_{u, u^{\prime}}\left(\rho_{y}\left(v^{\prime}\right)\right), v\right\rangle=\int_{G / Z}\left\langle\rho_{x}(u), \rho_{y}^{*}(v)\right\rangle\left\langle\rho_{x}\left(u^{\prime}\right), v^{\prime}\right\rangle^{-} d \dot{x}=\left\langle B_{u, u^{\prime}}\left(v^{\prime}\right), \rho_{y}^{*}(v)\right\rangle .
$$

Since the last term equals $\left\langle\rho_{y} B_{u, u^{\prime}}\left(v^{\prime}\right), v\right\rangle$ we conclude that $\left\langle B_{u, u^{\prime}}\left(\rho_{y}\left(v^{\prime}\right)\right), v\right\rangle=$ $\left\langle\rho_{y} B_{u, u^{\prime}}\left(v^{\prime}\right), v\right\rangle$, for all $y$ in $G$ and $u, v, u^{\prime}, v^{\prime}$ in $V$. It follows that $B_{u, u^{\prime}} \rho_{y}=\rho_{y} B_{u, u^{\prime}}$, for all $y$ in $G$ and $u, u^{\prime}$, in $V$. Hence, by Schur's Lemma, $B_{u, u^{\prime}}=\beta\left(u, u^{\prime}\right) I$ so that $\int_{G / Z}\left\langle\rho_{x}(u), v\right\rangle\left\langle\rho_{x}\left(u^{\prime}\right), v^{\prime}\right\rangle-d \dot{x}=\beta\left(u, u^{\prime}\right)\left\langle v^{\prime}, v\right\rangle$. On the other hand, since $\rho$ is unitary, $\left\langle\rho_{x}(u), v\right\rangle\left\langle\rho_{x}\left(u^{\prime}\right), v^{\prime}\right\rangle^{-}=\left\langle u, \rho_{x^{-1}}(v)\right\rangle\left\langle u^{\prime}, \rho_{x^{-1}}\left(v^{\prime}\right)\right\rangle^{-}$, so that

$$
\begin{aligned}
\left\langle B_{u, u^{\prime}}\left(v^{\prime}\right), v\right\rangle & =\int_{G / Z}\left\langle u, \rho_{x}{ }^{-1}(v)\right\rangle\left\langle u^{\prime}, \rho_{x^{-1}}\left(v^{\prime}\right)\right\rangle^{-} d \dot{x} \\
& =\int_{G / Z}\left\langle u, \rho_{x}(v)\right\rangle\left\langle u^{\prime}, \rho_{x}\left(v^{\prime}\right)\right\rangle^{-} d \dot{x},
\end{aligned}
$$

because of the unimodularity of $G / Z$. The last term equals

$$
\int_{G / Z}\left\langle\rho_{x}\left(v^{\prime}\right), u^{\prime}\right\rangle\left\langle\rho_{x}(v), u\right\rangle^{-} d \dot{x}=\beta\left(v^{\prime}, v\right)\left\langle u, u^{\prime}\right\rangle
$$

and therefore $\beta\left(u, u^{\prime}\right)\left\langle v^{\prime}, v\right\rangle=\beta\left(v^{\prime}, v\right)\left\langle u, u^{\prime}\right\rangle$, for all $u, v, u^{\prime}, v^{\prime}$ in $V$. Let $v^{\prime}=v$ and let $v$ be any fixed nonzero vector. Then $\beta\left(u, u^{\prime}\right)=\beta(v, v)\|v\|^{-2}\left\langle u, u^{\prime}\right\rangle$, i.e., $\beta\left(u, u^{\prime}\right)$ $=c_{\rho}\left\langle u, u^{\prime}\right\rangle$ where, as is easily seen, $c_{\rho}$ is a constant that does not depend upon $v$. Thus

$$
\int_{G / Z}\left\langle\rho_{x}(u), v\right\rangle\left\langle\rho_{x}\left(u^{\prime}\right), v^{\prime}\right\rangle-d \dot{x}=c_{\rho}\left\langle u, u^{\prime}\right\rangle\left\langle v^{\prime}, v\right\rangle
$$


Let $u^{\prime}=u, v^{\prime}=v$ and assume $\|u\|=\|v\|=1$ in the last equation. Then

$$
\int_{G / Z}\left|\left\langle\rho_{x}(u), v\right\rangle\right|^{2} d \dot{x}=c_{\rho}\|u\|^{2}\|v\|^{2}=c_{\rho} .
$$

By taking $u=v$ in this equation we see that $c_{\rho}>0$ because the integrand is nonnegative and has value 1 at $\dot{x}=\dot{1}$, since for $z \in Z(G)$ we have $\rho(z)=\lambda(z) I$, where $|\lambda(z)|=1$.

THEOREM 2.1. Let $G \in[Z]$ and $\rho$ be a continuous irreducible unitary representation of $G$ on the complex Hilbert space $V$. Then $\rho$ is finite-dimensional $\left({ }^{8}\right)$.

Proof. Let $\left\{v_{i} \mid i \in I\right\}$ be a maximal orthonormal family in $V$. For any finite subset $v_{1}, \ldots, v_{n}$ of this family we have

$$
\sum_{i=1}^{n}\left|\left\langle\rho_{x}\left(v_{i}\right), v_{1}\right\rangle\right|^{2} \leqq \sum_{i \in I}\left|\left\langle\rho_{x}\left(v_{i}\right), v_{1}\right\rangle\right|^{2},
$$

and since $\rho$ is as before and $\left\|v_{i}\right\|=1$ each summand in either of these sums, by the preceding Proposition, represents a function in $\mathscr{F}_{c}(G / Z)$. Hence, and by the above inequality,

$$
\begin{aligned}
\int_{G / Z} \sum_{i=1}^{n}\left|\left\langle\rho_{x}\left(v_{i}\right), v_{1}\right\rangle\right|^{2} d \dot{x} & =\sum_{i=1}^{n} \int_{G / Z}\left|\left\langle\rho_{x}\left(v_{i}\right), v_{1}\right\rangle\right|^{2} d \dot{x} \\
& =n c_{\rho} \leqq \int_{G / Z} \sum_{i}\left|\left\langle\rho_{x}\left(v_{i}\right), v_{1}\right\rangle\right|^{2} d \dot{x} .
\end{aligned}
$$

Since $\rho$ is unitary, $\left\{\rho_{x}\left(v_{i}\right)\right\}$, for every $x$, is also a maximal orthonormal family in $V$. By Parseval's Equation, $\left\|v_{1}\right\|^{2}=\sum_{i \in I}\left|\left\langle\rho_{x}\left(v_{i}\right), v_{1}\right\rangle\right|^{2}=1$. Hence $n c_{\rho} \leqq 1$. Since $c_{\rho}>0$, $n$ is bounded and the theorem follows.

In light of Theorem 2.1 we can reformulate the Proposition in a more realistic way.

THEOREM 2.2. Let $G \in[Z]$ and $\rho \in \mathscr{R}(G)$. For $\|u\|=\|v\|=1$ we have

$$
\int_{G / Z}\left|\left\langle\rho_{x}(u), v\right\rangle\right|^{2} d \dot{x}=d_{\rho}^{-1}
$$

Moreover, for each $u, v, u^{\prime}, v^{\prime}$ in $V_{\rho}$, we have

$$
\int_{G / Z}\left\langle\rho_{x}(u), v\right\rangle\left\langle\rho_{x}\left(u^{\prime}\right), v^{\prime}\right\rangle^{-} d \dot{x}=d_{\rho}^{-1}\left\langle u, u^{\prime}\right\rangle\left\langle v, v^{\prime}\right\rangle^{-} .
$$

Proof. Utilizing the method of proof of Theorem 2.1 we see that if $\left\{v_{1}, v_{2}, \ldots, v_{d_{\rho}}\right\}$ is an orthonormal basis for $V_{\rho}$, then for each $i=1, \ldots, d_{\rho}$ we have

$$
\int_{G / Z}\left|\left\langle\rho_{x}\left(v_{i}\right), v_{1}\right\rangle\right|^{2} d \dot{x}=c_{\rho}
$$

$\left.{ }^{8}\right)$ Theorem 2.1 as announced in [4] is false for $[F I A]^{-}$-groups, as was pointed out by Mautner [12]. 
and therefore

$$
d_{\rho} c_{\rho}=\sum_{i=1}^{d_{\rho}} \int_{G / Z}\left|\left\langle\rho_{x}\left(v_{i}\right), v_{1}\right\rangle\right|^{2} d \dot{x}=\int_{G / Z} \sum_{i=1}^{d_{\rho}}\left|\left\langle\rho_{x}\left(v_{i}\right), v_{1}\right\rangle\right|^{2} d \dot{x} .
$$

However, since $\rho$ is unitary, $\left\{\rho_{x}\left(v_{i}\right)\right\}$, for every $x$, is also an orthonormal basis for $V_{\rho}$. We may therefore conclude from Parseval's Equation and from the above that $d_{\rho} c_{\rho}=1$. The second assertion made in Theorem 2.2 is (3) of the Proposition. The next corollary follows directly.

Corollary 1. Let $v_{1}, \ldots, v_{d_{\rho}}$ be as above and let $\rho(x)=\left(\rho_{i j}(x)\right)$ be the matrix representation of $\rho$ relative to this basis. Then

$$
\int_{G / Z} \rho_{l i}(x)\left[\rho_{j k}(x)\right]^{-} d \dot{x}=d_{\rho}^{-1} \delta_{i k} \delta_{j l}
$$

and, in particular, $\int_{G / Z}\left|\chi_{\rho}\right|^{2} d \dot{x}=1$.

One can reformulate Corollary 1 in terms of integration over $G$.

COROLlARY 2. For any choice of the weighting function $w$, as constructed in Theorem 1.2, the system of functions $\left\{d_{\rho}^{1 / 2} w^{1 / 2} \rho_{i j} \mid i, j=1, \ldots, d_{\rho}\right\}$ is in $\mathscr{F}_{c_{0}}(G)$ and therefore in $L_{2}(G)$. Moreover, it is orthonormal in $L_{2}(G)$. And, in particular,

$$
\int_{G} w(x)\left|\chi_{\rho}(x)\right|^{2} d x=1 \text {. }
$$

The method of proof of the Proposition and of Theorem 2.1 has another consequence, namely

THEOREM 2.3. Let $G$ be an abstract group with center of finite index (i.e., $G \in[Z]$ ) and let $\rho$ be any irreducible unitary representation of $G\left({ }^{9}\right)$. Then $d_{o} \leqq[G: Z]$; in particular, these representations are of bounded degree.

Proof. We show, in general, that, for $G / Z$ compact and $\rho$ in $\mathscr{R}(G), d_{\rho} \leqq 1 / \mu_{G / Z}(i)$, where $\mathrm{i}$ is the identity of $G / Z$. This formula is meaningful only if $Z$ has finite index. In fact, via countable additivity, $\mu_{G / Z}(\mathrm{i})$ is positive if and only if $Z$ is of finite index in $G$; and in this case, $\mu_{G / Z}(\mathrm{i})=1 /[G: Z]$.

Continuing the proof we assume given $v$ in $V\left(=V_{\rho}\right)$ such that $\|v\|=1$. For $z$ in $Z(G)$ we have, as in the proof of the Proposition, $\rho(z)=\lambda(z) I$, where $|\lambda(z)|^{2}=1$. Hence $\left|\left\langle\rho_{z}(v), v\right\rangle\right|^{2}=1$. If $\phi_{i}$ denotes the characteristic function of $\{i\}$ then, since the function $x \rightarrow\left|\left\langle\rho_{x}(v), v\right\rangle\right|^{2}$ is in $\mathscr{F}_{c}(G / Z)$ (by the Proposition) and since the value of this function at $\dot{i}$ is 1 , we obtain

$$
\mu_{G / Z}(\mathrm{i})=\int_{G / Z} \phi_{i}\left|\left\langle\rho_{x}(v), v\right\rangle\right|^{2} d \dot{x} \leqq \int_{G / Z}\left|\left\langle\rho_{x}(v), v\right\rangle\right|^{2} d \dot{x}=d_{\rho}^{-1},
$$

by Theorem 2.2 . Therefore $d_{\rho} \leqq 1 / \mu_{G / Z}(\mathrm{i})$, and as was mentioned, if $[G: Z]$ is finite then the last quantity equals $[G: Z]$.

( $\left.{ }^{(}\right)$By Theorem 2.1, $\rho$ is finite-dimensional. 
REMARK. Actually, for discrete groups, a more general fact is known about representations of bounded degree for which we give a direct proof, and in addition obtain a sharpening of the estimate, in Theorem 2.3; we exploit this in Theorem 5.3 where we pursue this topic.

3. Separation and approximation theorems. A direct consequence of Theorem 2.1 together with the theorem of Gelfand-Raikov (see Naimark [15]) is that the set of all continuous finite-dimensional irreducible unitary representations of a $[Z]$-group $G$ separates the points of $G$ and, evidently, then so does $\mathscr{R}(G)$. Hence

THEOREM 3.1. If $G \in[Z]$ then $\mathscr{R}$ separates the points of $G$, i.e., $[Z]$-groups are $[M A P]$-groups $\left({ }^{10}\right)$.

In view of the last theorem together with Corollary 1 of Theorem 4.4 of [7], $[Z]$-groups satisfy the hypothesis of the Duality Theorem of Takahashi [18] which unifies the well-known duality theorems of Pontrjagin and Tannaka.

We shall see in $\S 5$, as a result of Theorem 5.5, that Theorem 3.1 can be derived without appealing to the results of Gelfand and Raikov.

THEOREM 3.2. Let $F$ be a compact subset of a [Z]-group $G$, let $f \in \mathscr{F}_{c}$, and let $\varepsilon$ be a positive number. Then there is a function $g$ in $\mathscr{F}_{r}$ satisfying $\|f-g\|_{F}<\varepsilon$.

Proof. It follows from the definition of $\mathscr{F}_{r}$ that it is a complex subspace of $\mathscr{F}_{u}$. Moreover, it is a subalgebra. To prove this it is sufficient to show that if $\rho$ and $\sigma \in \mathscr{R}$ and $\rho_{i j}$ and $\sigma_{k l}$ are coefficient functions of $\rho, \sigma$ respectively, then $\rho_{i j} \sigma_{k l} \in \mathscr{F}_{r}$. Now $\rho \otimes \sigma$ is a continuous finite-dimensional unitary representation of $G$ and hence is semisimple. Thus, for a suitable unitary operator $U$,

$$
\rho \otimes \sigma=U\left(\tau^{1} \oplus \tau^{2} \oplus \cdots \oplus \tau^{r}\right) U^{-1},
$$

where the $\tau^{i}$ are irreducible unitary representations. It is easy to see that, since each $\tau^{i}$ is unitarily equivalent to an element of $\mathscr{R}$, we may assume that, actually, $\tau^{i} \in \mathscr{R}$. From this and from the above, one then deduces that $\rho_{i j} \sigma_{k l} \in \mathscr{F}_{r}$. In addition to this the representation $\bar{\rho}$, defined by $\bar{\rho}(x)=[\rho(x)]^{-}$is also continuous, finitedimensional, unitary, and irreducible. Hence $\rho=U \sigma U^{-1}$, where $\sigma \in \mathscr{R}$ and $U$ is unitary. Hence $\mathscr{F}_{r}$ is closed under conjugation. Since $\mathscr{F}_{r}$ also contains the constants, Theorem 3.2 follows from Theorem 3.1 and the Stone-Weierstrass-Theorem.

For reasons of convenience we quote the following result.

Proposition 3.1 (BuRnside-Frobenius-SchUR). Let $G$ be an abstract group, $\rho^{1}, \rho^{2}, \ldots, \rho^{r}$ a set of mutually inequivalent irreducible finite-dimensional representations of $G$ and $\left\{\rho_{i j}^{k} \mid i, j=1, \ldots, \operatorname{deg} \rho^{k} ; k=1, \ldots, r\right\}$ the corresponding coordinate functions. Then the family $\left\{\rho_{i j}^{k}\right\}$ is linearly independent in $\mathscr{F}_{c}$.

$\left.{ }^{(10}\right)$ If $G$ is a connected [ $Z$ ]-group, then Theorem 3.1 in combination with the theorem of Freudenthal-Weil gives an alternative proof of Theorem 4.3 of [7]. 
This theorem was proven by Burnside for $r=1$ and by Frobenius and Schur in general (around the turn of the century); a proof may be found in van der Waerden [20].

Proposition 3.2 (AFTER PontrJagin [16]). If $G$ is any group the linear span of $\mathfrak{X}$ equals $\mathscr{F}_{r} \cap \mathscr{F}_{z}$.

Proof. Since characters are central representative functions, it is clear that the linear span of $\mathfrak{X}$ is contained in $\mathscr{F}_{r} \cap \mathscr{F}_{z}$.

Now suppose $f \in \mathscr{F}_{r} \cap \mathscr{F}_{z}$. Then

$$
f(x)=\sum_{k=1}^{r} \sum_{i, j=1}^{\operatorname{deg} \rho^{k}} c_{i j}^{k} \rho_{i j}^{k}(x), \quad \text { where } c_{i j}^{k} \in \mathbf{C} .
$$

Define $f_{k}(x)=\sum_{i, j=1}^{\operatorname{deg} \rho_{1}^{k}} c_{i j}^{k} \rho_{i j}^{k}(x)$, for $k=1, \ldots, r$. Then

$$
\begin{aligned}
f_{k}\left(y x y^{-1}\right) & =\sum_{i, j} c_{i j}^{k} \rho_{i j}^{k}\left(y x y^{-1}\right)=\sum_{i, j} c_{i j}^{k} \sum_{l, m} \rho_{i l}^{k}(y) \rho_{l m}^{k}(x) \rho_{m j}^{k}\left(y^{-1}\right) \\
& =\sum_{l, m}\left(\sum_{i, j} c_{i j}^{k} \rho_{i l}^{k}(y) \rho_{m j}^{k}\left(y^{-1}\right)\right) \rho_{l m}^{k}(x) .
\end{aligned}
$$

Now let $d_{l m}^{k}(y)=\sum_{i, j} c_{i j}^{k} \rho_{i l}^{k}(y) \rho_{m j}^{k}\left(y^{-1}\right)$. Then, since $f$ is central,

$$
f(x)=f\left(y x y^{-1}\right)=\sum_{k} f_{k}\left(y x y^{-1}\right)=\sum_{k=1}^{r} \sum_{l, m=1}^{\operatorname{deg} \rho^{k}} d_{l m}^{k}(y) \rho_{l m}^{k}(x) .
$$

Hence, and by Proposition 3.1, $c_{l m}^{k}=d_{l m}^{k}(y)$, for each triple $(l, m, k)$, i.e.,

$$
\sum_{i, j} c_{i j}^{k} \rho_{i l}^{k}(y) \rho_{m j}^{k}\left(y^{-1}\right)=c_{l m}^{k}
$$

and hence $f_{k}\left(y x y^{-1}\right)=\sum_{l, m} c_{l m}^{k} \rho_{l m}^{k}(x)=f_{k}(x)$, so that each $f_{k}$ is central. The proof will be complete if we can show that if $g=\sum_{i, j=1}^{\operatorname{deg} \rho} c_{i j} \rho_{i j}$, where $\rho$ is irreducible and $g$ is central, then $g=\lambda \chi_{\rho}$, where $\lambda \in \mathbf{C}$. Since $g$ is central, it follows that $g(x y)=g(y x)$, for each $x, y$ in $G$. Hence

$$
\sum_{i, j, k} c_{i j} \rho_{i k}(x) \rho_{k j}(y)=\sum_{i^{l}, j^{l}, k^{l}} c_{i^{l} j^{l}} \rho_{i^{l} k^{l}}(y) \rho_{k^{l} j^{l}}(x),
$$

so that, for fixed $y$,

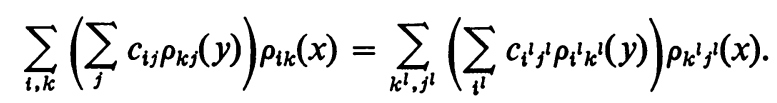

Applying Proposition 3.1 to this we obtain

$$
\sum_{j} c_{i j} \rho_{k j}(y)=\sum_{i^{l}} c_{i}{ }^{l} k \rho_{i}{ }^{l_{i}}(y), \quad \text { or } \sum_{j} c_{i j} \rho_{k j}(y)=\sum_{j} \rho_{j i}(y) c_{j k},
$$

for each pair $(i, k)$. If we denote the matrix $\left(c_{i j}\right)$ by $C$ the last equation states that $\rho_{y} C^{t}=C^{t} \rho_{y}$, for each $y$ in $G$, where $C^{t}$ is the transpose of $C$. Since $\rho$ is irreducible, we have $C^{t}=\lambda I=C$, by Schur's Lemma. 
THEOREM 3.3. Let $F$ be a compact subset of the [Z]-group $G, f \in \mathscr{F}_{c} \cap \mathscr{F}_{z}$, and $\varepsilon$ be a positive number. Then there exist $\rho^{k}$ in $\mathscr{R}$ and $c_{k}$ in $\mathbf{C}(k=1, \ldots, r)$ such that $\left\|f-\sum_{k=1}^{r} c_{k} \chi_{\rho}\right\|_{F}<\varepsilon$.

Proof. Replacing, if necessary, $F$ by the compact set $G \square F$ which contains $F$ and is invariant, we may assume that $F$ is invariant. By Theorem 3.2, there exists a function $g$ in $\mathscr{F}_{r}$ satisfying $\|f-g\|_{F}<\varepsilon$. Assume for the moment that $f \in \mathscr{F}_{u}$. Then, since $g \in \mathscr{F}_{u}, f-g \in \mathscr{F}_{u}$. We apply \# (Theorem 1.1) and conclude that $(f-g)^{\#}$ $=f^{\#}-g^{\#}=f-g^{\#}$, since $f \in \mathscr{F}_{z}$. Moreover, $g^{\#} \in \mathscr{F}_{r} \cap \mathscr{F}_{z}$. By Proposition 3.2, $g^{\#}=\sum_{k=1}^{r} c_{k} \chi_{\rho^{k}}$. Thus $(f-g)^{\#}=f-\sum_{k=1}^{r} c_{k} \chi_{\rho^{k}}$, so that by Theorem 1.1,

$$
\left\|f-\sum_{k=1}^{r} c_{k} \chi_{\rho}\right\|_{F}=\left\|(f-g)^{\#}\right\|_{F} \leqq\|f-g\|_{F}<\varepsilon .
$$

Now let $f$ be any element of $\mathscr{F}_{c}$. By Urysohn's Lemma, choose a function $f_{1} \in \mathscr{F}_{c_{0}}$ such that $\left(f_{1}\right)_{F}=1$ and let $f_{2}=f f_{1}$. Evidently, $f_{2} \in \mathscr{F}_{c_{0}}$. Since $f_{2}$ has compact support, it is uniformly continuous, and the part of the theorem already proven applies to $f_{2}$. Since $f_{F}=\left(f_{2}\right)_{F}$, the proof is complete.

THEOREM 3.4. If $G \in[Z]$ then $\mathfrak{X}$ separates the conjugacy classes of $G$.

Proof. Let $x$ and $y$ be nonconjugate elements of $G$ (if such elements exist) and $>x<$ and $>y<$ their conjugacy classes. Since $G \in[Z],>x<$ and $>y<$ are compact (see [7], Theorem 4.2). Since $>x<$ and $>y<$ are disjoint and $G$ is a locally compact Hausdorff space there is a compact neighborhood $U$ of $>x<$ disjoint from $>y<$. Hence there is a function $f$ in $\mathscr{F}_{c}$ such that $1 \geqq f \geqq 0, f_{>x<}=1$ and Supp $f \subseteq U$. Then $f$ is uniformly continuous and $f_{>y<}=0$. Applying \# we find that $f^{\#}(x)=1$ and

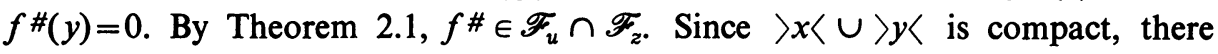
exists (by Theorem 3.3) a linear combination of characters such that

$$
\left\|f^{\#}-\sum_{k=1}^{r} c_{k} \chi_{\rho}\right\|_{>x\langle u\rangle y<}<1 / 2 .
$$

Then evidently, for some $k, \chi_{\rho}^{k}(x) \neq \chi_{\rho}(y)$. This completes the proof.

4. The character formula, irreducibility criteria, and an application.

LEMMA 4.1. Let $G$ be any group and $\rho$ and $\sigma$ irreducible unitary representations of $G$. If $\rho$ and $\sigma$ are equivalent in $G L(n, C)$ then they are unitarily equivalent.

In fact, the following more general result is well-known: Let $\rho$ and $\sigma$ be unitary representations of $G$ on a Hilbert space. If $\rho$ and $\sigma$ are equivalent by a bounded operator then they are unitarily equivalent.

THEOREM 4.1. For any group $G$, the map $\rho \rightarrow \chi_{\rho}$ from $\mathscr{R}$ to $\mathfrak{X}$ is bijective.

Proof. Suppose $\chi_{\rho}(x)=\chi_{\sigma}(x)$, for all $x$ in $G$. For $x=1$, this gives $d_{\rho}=d_{\sigma}$; denote this number by $n$. Then, for all $x$ in $G, \sum_{i=1}^{n} \rho_{i i}(x)=\sum_{i=1}^{n} \sigma_{i i}(x)$. If $\rho$ and $\sigma$ are 
unequivalent then this constitutes a nontrivial dependence relation and contradicts Proposition 3.1. Hence $\rho$ and $\sigma$ are equivalent in $G L(n, \mathbf{C})$ and therefore, by Lemma 4.1, they are unitarily equivalent.

ThEOREM 4.2. Let $G \in[Z]$ and $\rho \in \mathscr{R}$. Then $\chi_{\rho}$ is almost periodic and satisfies the functional equation

$$
f(s) f(t)=f(1) \int_{G / Z} f\left(x s x^{-1} t\right) d \dot{x} \quad(s, t \in G) .
$$

Conversely, if $f$ is any nontrivial almost periodic function satisfying (*) then

$$
\frac{f(s)}{f(1)}=\frac{\chi_{\rho}(s)}{\chi_{\rho}(1)}
$$

for a unique $\rho$ in $\mathscr{R}$.

Before giving the proof we remark that Theorem 4.2 generalizes the classical character formula for compact groups. (See Weil [21].) For (as was stated in \$1) if $G$ is compact and $f \in \mathscr{F}_{c}(G / Z)$ then $\int_{G} f(x) d x=\int_{G / Z} f(\dot{x}) d \dot{x}$, and elements of $\mathscr{F}_{c}(G)$ are almost periodic. In addition, Theorem 4.2 shows that in the case of an abelian group $G$ a function $f$ in $\mathscr{F}_{c}(G)$ is a group character if and only if it is almost periodic and $f(f(1)$ is a continuous homomorphism of $G$ into the multiplicative group of $\mathbf{C}$.

Proof of Theorem 4.2. We extend the \# operator (as defined in Theorem 1.1) to representations by defining

$$
\rho^{\#}(s)=\int_{G / Z} \rho\left(x s x^{-1}\right) d \dot{x} .
$$

This makes sense because, for $s$ fixed, $\rho\left(x s x^{-1}\right)$ is a continuous operator-valued function on $G / Z$. For $t$ in $G$, we have

$$
\rho(t) \rho^{\#}(s) \rho(t)^{-1}=\int_{G / Z} \rho(t) \rho\left(x s x^{-1}\right) \rho(t)^{-1} d \dot{x}=\int_{G / Z} \rho\left((t x) s(t x)^{-1}\right) d \dot{x} .
$$

By the invariance of the Haar integral this equals $\int_{G / Z} \rho\left(x s x^{-1}\right) d \dot{x}$, so that $\rho(t) \rho^{\#}(s)=\rho^{\#}(s) \rho(t)$, for all $s, t$ in $G$. Since $\rho$ is irreducible, it follows that $\rho^{\#}(s)=\lambda(s) I$ and therefore $\lambda(s)=(\operatorname{tr} \rho \#(s)) / d_{\rho}$. On the other hand,

$$
\operatorname{tr} \rho^{\#}(s)=\sum_{i=1}^{d_{\rho}} \int_{G / Z} \rho_{i i}\left(x s x^{-1}\right) d \dot{x}=\int_{G / Z} \chi_{\rho}\left(x s x^{-1}\right) d \dot{x}=\chi_{\rho}^{\#(s) . ~}
$$

Since $\chi_{\rho}$ is central, it follows from this and from Theorem 1.1 that $\lambda(s)=\chi_{\rho}(s) d_{\rho}^{-1}$ and therefore

$$
\int_{G / Z} \rho\left(x s x^{-1}\right) d \dot{x}=\chi_{\rho}(s) d_{\rho}^{-1} I
$$


It now follows that, for each $t$ in $G$,

$$
\left(\int_{G / Z} \rho\left(x s x^{-1}\right) d \dot{x}\right) \rho(t)=\int_{G / Z} \rho\left(x s x^{-1} t\right) d \dot{x}=\chi_{\rho}(s) d_{\rho}^{-1} \rho(t)
$$

and, from this, that $\chi_{\rho}$ satisfies (*); simply take the trace in the last equation. Clearly, $\chi_{\rho}$ is almost periodic.

Conversely, suppose $f$ satisfies $(*)$. We first show that then $f(1) \neq 0$. For if $f(1)=0$ then $f(s) f(t)=0$, for all $s, t$ in $G$. If $f(t) \neq 0$, for some $t$, then the last statement implies that $f(s)=0$, for all $s$ in $G$, a contradiction to $f(t) \neq 0$. Thus, if $t=1$, we obtain $f(s)=f^{\#}(s)$, i.e., $f=f^{\#}$, so that $f$ is central (Theorem 1.1).

Before we proceed with the proof of the converse we note a few well-known facts concerning almost periodic functions. Let $G^{\alpha}$ be the Bohr group of $G$ and $\alpha: G \rightarrow G^{\alpha}$ the canonical continuous monomorphism (Theorem 3.1) of $G$ onto a dense subgroup of $G^{\alpha}$. Denote by $\phi^{\alpha}$ the functions on $G^{\alpha}$ associated with almost periodic functions $\phi$ on $G$, by $\mathscr{F}_{a}(G)$ the continuous almost periodic complex functions on $G$, and (as defined in $\S 1$ ) by $\mathscr{F}_{c}\left(G^{\alpha}\right)$ the continuous complex functions on $G^{\alpha}$. Then the following holds:

(a) If $\phi$ is central then so is $\phi^{\alpha}$.

(b) The map $\phi \rightarrow \phi^{\alpha}$ is an algebra homomorphism from $\mathscr{F}_{a}(G)$ to $\mathscr{F}_{c}\left(G^{\alpha}\right)$ which commutes with complex conjugation.

(c) The finite-dimensional continuous unitary representations $\rho$ of $G$ and $\rho^{\alpha}$ of $G^{\alpha}$ are in bijective correspondence.

(d) In (c) irreducible representations and unitary equivalence classes correspond.

(e) If $\rho$ and $\rho^{\alpha}$ correspond then $\left(\chi_{\rho}\right)^{\alpha}=\chi_{\rho}^{\alpha}$.

For facts concerning the Bohr group see [21].

Now, continuing the proof, we denote by $\int_{*} \phi(t) d t$ the invariant integral on the algebra $\mathscr{F}_{a}(G)$, i.e., $\int_{*} \phi(t) d t=\int_{G^{\alpha}} \phi^{\alpha}(u) d u$. If $\phi_{1}$ and $\phi_{2} \in \mathscr{F}_{a}(G)$, denote $\int_{*} \phi_{1}(t)\left(\phi_{2}(t)\right)^{-} d t$ by $\left\langle\phi_{1}, \phi_{2}\right\rangle_{*}$. Then $\langle,\rangle_{*}$ is an inner product on $\mathscr{F}_{a}(G)$. We require a lemma.

LEMMA 4.2. If $\phi$ is a central function in $\mathscr{F}_{a}(G)$ and $\left\langle\phi, \chi_{\rho}\right\rangle_{*}=0$, for every $\rho$ in $\mathscr{R}$, then $\phi=0$.

Proof. By (a) above, $\phi^{\alpha}$ is central. Hence, and because $G^{\alpha}$ is compact,

$$
\left\|\phi^{\alpha}\right\|_{2}^{2}=\sum_{\rho^{\alpha} \in \mathscr{R}_{\left(G^{\alpha}\right)}}\left|\left\langle\phi^{\alpha}, \chi_{\rho^{\alpha}}\right\rangle\right|^{2} .
$$

However, since $\mathscr{R}\left(G^{\alpha}\right)$ is in bijective correspondence with $\mathscr{R}(G)$ (by (c) above), since $\chi_{\rho}^{\alpha}=\left(\chi_{\rho}\right)^{\alpha}$ and since $\left\langle\phi, \chi_{\rho}\right\rangle_{*}=\int_{G^{\alpha}} \phi^{\alpha}(u)\left(\chi_{\rho^{\alpha}}(u)\right)^{-} d u$ (by (b)), it follows that

$$
\left\|\phi^{\alpha}\right\|_{2}^{2}=\sum_{\rho \in \mathscr{R}(G)}\left|\left\langle\phi, \chi_{\rho}\right\rangle_{*}\right|^{2} .
$$

Now, by assumption, $\left\langle\phi, \chi_{\rho}\right\rangle_{*}=0$, for all $\rho$. Therefore, $\left\|\phi^{\alpha}\right\|_{2}^{2}=0$, and since $\phi^{\alpha}$ is continuous, this implies that $\phi^{\alpha}=0$, so that $\phi=0$. 
The proof of the theorem will be complete if we can show that, for every $\rho$ in $\mathscr{R}$,

$$
\frac{f(s)}{f(1)}\left\langle f, \chi_{\rho}\right\rangle_{*}=\frac{\chi_{\rho}(s)}{\chi_{\rho}(1)}\left\langle f, \chi_{\rho}\right\rangle_{*} \quad(s \in G) .
$$

For in this case, since $f$ is central and $f \neq 0$, there is some $\rho$ in $\mathscr{R}$ such that $\left\langle f, \chi_{\rho}\right\rangle_{*} \neq 0$ (Lemma 4.2). Hence, for that $\rho, f(s) / f(1)=\chi_{\rho}(s) / \chi_{\rho}(1)$. If also $\chi_{\sigma}(s) / \chi_{\sigma}(1)$ $=f(s) / f(1)$, where $\sigma \in \mathscr{R}$, then one sees easily from Proposition 3.1 and Lemma 4.1 that $\rho=\sigma$.

In order to prove $(* * *)$ we consider $\int_{G / z} \int_{*} f\left(x s x^{-1} t\right)\left(\chi_{\rho}(t)\right)^{-} d \dot{x} d t$. By the Fubini Theorem this is $\int_{*}\left(\chi_{\rho}(t)\right)^{-}\left(\int_{G / Z} f\left(x s x^{-1} t\right) d \dot{x}\right) d t$. But since $f(1) \neq 0$, this equals, via (*),

$$
\int_{*}\left(\chi_{\rho}(t)\right)^{-} \frac{f(s) f(t)}{f(1)} d t=\frac{f(s)}{f(1)} \int_{*} f(t)\left(\chi_{\rho}(t)\right)^{-} d t=\frac{f(s)}{f(1)}\left\langle f, \chi_{\rho}\right\rangle_{*},
$$

where the second equation follows from the remarks made about the Bohr group.

On the other hand,

$$
\int_{G / Z} \int_{*} f\left(x s x^{-1} t\right)\left(\chi_{\rho}(t)\right)^{-} d \dot{x} d t=\int_{G / Z}\left(\int_{*} f\left(x s x^{-1} t\right)\left(\chi_{\rho}(t)\right)^{-} d t\right) d \dot{x} .
$$

Keeping in mind that $\int_{*}$ is invariant under left translation we have

$$
\int_{*} f\left(x s x^{-1} t\right)\left(\chi_{\rho}(t)\right)^{-} d t=\int_{*} f(t)\left(\chi_{\rho}\left(x s^{-1} x^{-1} t\right)\right)^{-} d t .
$$

Since $\left(\chi_{\rho}(y)\right)^{-}=\chi_{\rho}\left(y^{-1}\right)$, it follows that $\left(\chi_{\rho}\left(x s^{-1} x^{-1} t\right)\right)^{-}=\chi_{\rho}\left(t^{-1} x s x^{-1}\right)$; and since $\chi_{\rho}$ is central, we get $\chi_{\rho}\left(t^{-1} x s x^{-1}\right)=\chi_{\rho}\left(x s x^{-1} t^{-1}\right)$. Therefore

$$
\int_{*} f\left(x s x^{-1} t\right)\left(\chi_{\rho}(t)\right)^{-} d t=\int_{*} f(t) \chi_{\rho}\left(x s x^{-1} t^{-1}\right) d t
$$

and hence

$$
\begin{aligned}
\int_{G / Z} \int_{*} f\left(x s x^{-1} t\right)\left(\chi_{\rho}(t)\right)^{-} d \dot{x} d t & =\int_{G / Z}\left(\int_{*} f(t) \chi_{\rho}\left(x s x^{-1} t^{-1}\right) d t\right) d \dot{x} \\
& =\int_{*} f(t)\left(\int_{G / Z} \chi_{\rho}\left(x s x^{-1} t^{-1}\right) d \dot{x}\right) d t .
\end{aligned}
$$

By the first part of the present theorem, $\chi_{\rho}$ satisfies (*), so that the last term equals

$$
\int_{*} f(t) \frac{\chi_{\rho}(s) \chi_{\rho}\left(t^{-1}\right)}{\chi_{\rho}(1)} d t
$$

Hence

$$
\frac{\chi_{\rho}(s)}{\chi_{\rho}(1)} \int_{*} f(t)\left(\chi_{\rho}(t)\right)^{-} d t=\frac{\chi_{\rho}(s)}{\chi_{\rho}(1)}\left\langle f, \chi_{\rho}\right\rangle_{*}
$$

The proof of Theorem 4.2 is now complete. 
We now derive irreducibility criteria for finite-dimensional continuous unitary representations.

THEOREM 4.3. Let $G$ be $a[Z]$-group and $\rho$ a finite-dimensional continuous unitary representation of $G$. Then a necessary and sufficient condition for $\rho$ to be irreducible is that either conditions (1) and (2) or conditions (1) and (3) hold, where (1), (2), and (3) are as follows:

$$
\int_{G / Z} \rho\left(x y x^{-1}\right) d \dot{x}=\frac{\chi_{\rho}(y)}{\chi_{\rho}(1)} I_{d_{\rho}} \quad(y \in G) .
$$

(2) $\left|\chi_{\rho}\right|^{2}$ is a function on $G / Z$ and $\int_{G / Z}\left|\chi_{\rho}(x)\right|^{2} d \dot{x}=1$.

(3) $\left|\left\langle\rho_{x}(v), v\right\rangle\right|^{2}$ is a function on $G / Z$ and, for $\|v\|=1$,

$$
\int_{G / Z}\left|\left\langle\rho_{x}(v), v\right\rangle\right|^{2} d \dot{x}=d_{\rho}^{-1}
$$

Before we give the proof we remark that the left-hand side of (1) is meaningful; it is simply $\rho^{\#}(y)$, as defined in the proof of Theorem 4.2.

Proof. Assume first that $\rho$ is irreducible. Then (1) is simply equation (**) derived in the course of the proof of the character formula (Theorem 4.2). Also (2) and (3) have been verified in Theorem 2.2 and its corollaries.

Now assume first that $\rho$ is unitary and that (1) holds. Then, by the semisimplicity of $\rho$, we have $\rho=\sum_{i=1}^{r} \oplus \rho^{i}$, where the $\rho^{i}$ are irreducible unitary representations, and $V_{\rho}=\sum_{i=1}^{r} \oplus V_{i}$, an orthogonal direct sum, where $V_{i}$ is invariant under $\rho$ and $\left(\rho^{i}\right)_{x}=\left(\rho_{x}\right)_{v_{i}}$, for $x \in G$ and $i=1, \ldots, r$. In terms of a suitable orthonormal basis for $V_{\rho}$ we have $\rho(x)=\rho^{1}(x) \oplus \cdots \oplus \rho^{r}(x)$. Hence $\int_{G / Z} \rho\left(x y x^{-1}\right) d \dot{x}$ is a matrix made up of blocks down the diagonal of the form $\int_{G / Z} \rho^{i}\left(x y x^{-1}\right) d \dot{x}$. Since $\rho^{i}$ is irreducible and unitary, the first half of the present theorem implies that

$$
\int_{G / Z} \rho^{i}\left(x y x^{-1}\right) d \dot{x}=d_{\rho^{i}}^{-1} \chi_{\rho^{i}}(y) I_{\operatorname{deg} \rho^{i}}
$$

On the other hand, applying (1), we see that $\chi_{\rho^{\prime}}(y) d_{\rho^{i}}^{-1}=\chi_{\rho}(y) d_{\rho}^{-1}$, for each $i=1, \ldots, r$. Therefore, $\chi_{\rho^{i}}(y) d_{\rho^{i}}^{-1}=\chi_{\rho^{\prime}}(y) d_{\rho^{j}}^{-1}$, for all $i, j$. For $i \neq j$, this is a nontrivial dependence relation, a contradiction to Proposition 3.1 unless $\rho^{i}$ and $\rho^{j}$ are equivalent; but then they are unitarily equivalent.

For each $i(i=1, \ldots, r)$ let $A_{i}: V_{1} \rightarrow V_{i}$ be a unitary operator satisfying $A_{i}\left(\rho^{1}\right)_{x}=\left(\rho^{i}\right)_{x} A_{i}$, for all $x$ in $G$. (Here, $A_{1}=I$.) Let $w$ be any vector in $V_{1}$ of norm 1 , and let $u_{i}=A_{i}(w)$. Then $u_{i} \in V_{i},\left\|u_{i}\right\|=1$, and

$$
\left\langle\rho_{x}^{i}\left(u_{i}\right), u_{i}\right\rangle=\left\langle\rho_{x}^{i}\left(A_{i}(w)\right), A_{i} w\right\rangle=\left\langle A_{i}\left(\rho_{x}^{1}(w)\right), A_{i}(w)\right\rangle=\left\langle\rho_{x}^{1}(w), w\right\rangle,
$$

because $A_{i}$ is unitary. Now denote $\sum_{i=1}^{r} \oplus u_{i}$ by $u$, so that $u \in V_{\rho},\|u\|^{2}=\sum_{i}\left\|u_{i}\right\|^{2}=r$, and therefore $u \neq 0$. Since $\rho_{x}(u)=\sum \oplus \rho_{x}^{i}\left(u_{i}\right)$, it follows that

$$
\left\langle\rho_{x}(u), u\right\rangle=\left\langle\sum_{i} \rho_{x}^{i}\left(u_{i}\right), \sum_{j} u_{j}\right\rangle=\sum_{i}\left\langle\rho_{x}^{i}\left(u_{i}\right), u_{i}\right\rangle,
$$


since $V_{i}$ is orthogonal to $V_{j}$, if $i \neq j$, and each $V_{i}$ is invariant under $\rho^{i}$. Consequently,

$$
\left|\left\langle\rho_{x}(u), u\right\rangle\right|^{2}=\sum_{i, j=1}^{r}\left\langle\rho_{x}^{i}\left(u_{i}\right), u_{i}\right\rangle\left\langle\rho_{x}^{j}\left(u_{j}\right), u_{j}\right\rangle^{-}
$$

and therefore

$$
\int_{G / Z}\left|\left\langle\rho_{x}(u), u\right\rangle\right|^{2} d \dot{x}=\sum_{i, j} \int_{G / Z}\left\langle\rho_{x}^{i}\left(u_{i}\right), u_{i}\right\rangle\left\langle\rho_{x}^{j}\left(u_{j}\right), u_{j}\right\rangle^{-} d \dot{x} .
$$

But

$$
\int_{G / Z}\left\langle\rho_{x}^{i}\left(u_{i}\right), u_{i}\right\rangle\left\langle\rho_{x}^{j}\left(u_{j}\right), u_{j}\right\rangle^{-} d \dot{x}=\int_{G / Z}\left\langle\rho_{x}^{1}(w), w\right\rangle\left\langle\rho_{x}^{1}(w), w\right\rangle^{-} d \dot{x},
$$

which equals $c_{\rho^{1}}$ (by Theorem 2.2), since $\rho^{1}$ is an irreducible unitary representation and $\|w\|=1$. Therefore

$$
\int_{G / Z}\left|\left\langle\rho_{x}(u), u\right\rangle\right|^{2} d \dot{x}=\sum_{i, j} c_{\rho^{1}}=r^{2} c_{\rho^{1}} .
$$

Now, for any $\lambda$ in $\mathbf{R},\left\langle\rho_{x}(\lambda u), \lambda u\right\rangle=|\lambda|^{2}\left\langle\rho_{x}(u), u\right\rangle$, so that

and therefore

$$
\left|\left\langle\rho_{x}(\lambda u), \lambda u\right\rangle\right|^{2}=|\lambda|^{4}\left|\left\langle\rho_{x}(u), u\right\rangle\right|^{2}
$$

$$
\int_{G / Z}\left|\left\langle\rho_{x}(\lambda u), \lambda u\right\rangle\right|^{2} d \dot{x}=|\lambda|^{4} \int_{G / Z}\left|\left\langle\rho_{x}(u), u\right\rangle\right|^{2} d \dot{x} .
$$

Since $u \neq 0$, we may take $\lambda=1 /\|u\|$. Let $v=\lambda u$. Then

$$
\int_{G / Z}\left|\left\langle\rho_{x}(v), v\right\rangle\right|^{2} d \dot{x}=\|u\|^{-4} \int_{G / Z}\left|\left\langle\rho_{x}(u), u\right\rangle\right|^{2} d \dot{x}=r^{2} c_{\rho^{1}} / r^{2}=c_{\rho^{1}},
$$

since $\|u\|^{2}=r$.

Now assume that (3) holds. Then, since $\|v\|=1$, we have

$$
\int_{G / Z}\left|\left\langle\rho_{x}(v), v\right\rangle\right|^{2} d \dot{x}=d_{\rho}^{-1} \text {. }
$$

Thus $c_{\rho^{1}}=d_{\rho}^{-1}$. Taking into account that $c_{\rho^{1}}=d_{\rho^{1}}^{-1}$ (since $\rho^{1}$ is irreducible), and that $d_{\rho}=r d_{\rho^{1}}$, we see that $r=1$, so that $\rho$ is irreducible.

Now assume that (1) and (2) hold. Then, by the proof of the part of the converse involving condition (1) we have $\chi_{\rho}(x)=r \chi_{\rho}{ }^{1}(x)$, for all $x$ in $G$. Hence

$$
\left|\chi_{\rho}(x)\right|^{2}=r^{2}\left|\chi_{\rho}(x)\right|^{2},
$$

so that

$$
\int_{G / Z}\left|\chi_{\rho}(x)\right|^{2} d \dot{x}=1=r^{2} \int_{G / Z}\left|\chi_{\rho}^{1}(x)\right|^{2} d \dot{x} .
$$

Since $\rho^{1}$ is irreducible, it follows from the first part of the present theorem that $\int_{G / Z}\left|\chi_{\rho}{ }^{1}(x)\right|^{2} d \dot{x}=1$. Hence $r=1$, and $\rho$ is indeed irreducible. 
REMARK. For example it follows from Theorem 4.3 (via the Fubini Theorem) that if $\rho$ and $\sigma$ are continuous irreducible unitary representations of $G$ and $H$, respectively, and $G, H \in[Z]$ then $\rho \otimes \sigma$ is a continuous irreducible unitary representation of $G \times H$. Although this result is true for general locally compact groups, it is interesting that for $[Z]$-groups its proof can be made to depend upon integration.

The proof of Theorem 4.3 also yields the following:

COROLLARY. Under the hypothesis of Theorem $4.3, \rho$ is a primary representation if and only if it satisfies (1) of Theorem 4.3.

The following proposition generalizes a result of I. M. Isaacs and D. S. Passman [9] while relying on their method of proof as well as a theorem of D. Suprunenko( $\left.{ }^{11}\right)$.

Proposition 4.1. Let $G$ be a locally compact group which is nilpotent of class 2 and $\rho \in \mathscr{R}(G)$. If $\rho$ is faithful on $Z(G)$ then $\rho$ is faithful and $[G: Z]$ is finite; in fact $[G: Z]=d_{\rho}^{2}$.

Proof. We first show Supp $\chi_{\rho} \subseteq Z$. If $x \notin Z$ then there is a $y \in G$ such that $y x y^{-1} x^{-1} \neq 1$. But this commutator $\in G^{\prime} \subseteq Z$. Hence $y x y^{-1}=z x$ where $z \in Z$ and $z \neq 1$. It follows that $\chi_{\rho}\left(y x y^{-1}\right)=\chi_{\rho}(x)=\chi_{\rho}(z x)$. On the other hand, since $\rho$ is irreducible, $\rho(z x)=\lambda(z) \rho(x)$ where $\lambda \in Z^{\wedge}$. Thus $\chi_{\rho}(z x)=\lambda(z) \chi_{\rho}(x)$. Since $\chi_{\rho}(z)=d_{\rho} \lambda(z)$ we have $\chi_{\rho}(z x)=d_{\rho}^{-1} \chi_{\rho}(z) \chi_{\rho}(x)$. If $\chi_{\rho}(x) \neq 0$ then $\chi_{\rho}(z) d_{\rho}^{-1}=1$. It is well known and easy to see that $\operatorname{Ker} \rho=\left\{x \mid x \in G, \chi_{\rho}(x)=d_{\rho}\right\}$. It follows that $z=1$ since $\rho_{z}$ is faithful, a contradiction.

It follows from the above that $\operatorname{Ker} \rho \subseteq Z$ and hence is trivial. Thus $\rho$ is faithful. A theorem of Suprunenko tells us that $[G: Z]<\infty$. By Theorem 4.3 (since $G$ is a [Z]-group), $\int_{G / Z}\left|\chi_{\rho}(x)\right|^{2} d \dot{x}=1$. However, for $x \notin Z$ we have $\left|\chi_{\rho}(x)\right|^{2}=0$, so that $1=\int_{G / Z} \phi_{\mathrm{i}}\left|\chi_{\rho}(x)\right|^{2} d \dot{x}=\int_{G / Z}\left|\chi_{\rho}(z)\right|^{2} d \dot{x}$. Since $\left|\chi_{\rho}(z)\right|^{2} \equiv d_{\rho}$ on $Z$ we have

$$
1=d_{\rho}^{2} \mu_{G / Z}(\mathrm{i})=d_{\rho}^{2}[G: Z]^{-1},
$$

since $[G: Z]$ is finite.

REMARK. Although it is true that in a nilpotent group any nontrivial normal subgroup intersects the center nontrivially, so that the first assertion of the Proposition follows directly, in order to verify the second one it is necessary to show Supp $\chi_{\rho} \subseteq Z$.

The following corollary gives a method for constructing groups which satisfy many finiteness conditions but are not $[M A P]$-groups.

COROLlaRY. Let $G$ be an infinite group with the property that $G^{\prime}=Z(G)$ has order $p$, a prime. Then each $\rho \in \mathscr{R}(G)$ annihilates $Z(G)$.

(11) We are indebted to Professor O. Kegel for calling to our attention this theorem which appears in Soluble and nilpotent linear groups, by D. Suprunenko, Amer. Math. Soc. Translations of Math. Monographs, vol. 9, 1963. 
Proof. If there is a $\rho \in \mathscr{R}(G)$ which doesn't annihilate $Z(G)$ then $\rho_{Z}$ is faithful and hence $[G: Z]<\infty$. Since $Z$ is finite then so is $G$, a contradiction.

5. Extension of group characters; representations of bounded degree. We begin $\S 5$ with some definitions.

Definition 1. If $H$ is an abelian topological group and $\chi: H \rightarrow \mathrm{T}$ is a continuous homomorphism with values in the circle group (written multiplicatively) we call $\chi$ a group character of $H$, in order to distinguish from our previous use of the term "character."

Definition 2. Let $G$ be a topological group, $H$ an abelian subgroup, $\chi$ a group character of $H$, and $\rho$ a finite-dimensional continuous representation of $G$. We say $\rho$ extends $\chi$ if $\rho(y)=\chi(y) I_{d_{\rho}}$, for all $y$ in $H$.

THEOREM 5.1. Let $G$ be an [MAP]-group and $K$ a compact subgroup of $G$. Then each continuous unitary irreducible representation of $K$ is (up to unitary equivalence) an irreducible component of the restriction $\rho_{K}$ of a finite-dimensional continuous irreducible unitary representation $\rho$ of $G$.

Proof. For each $\rho$ in $\mathscr{R}(G), \rho_{K}$ is a continuous finite-dimensional unitary representation of $K$. By semisimplicity we have

$$
\rho_{K}=\sigma_{1}^{\rho} \oplus \rho_{2}^{\rho} \oplus \cdots \oplus \sigma_{r_{\rho}}^{\rho}
$$

where $\sigma_{i}^{o}$ is a continuous irreducible unitary representation of $K$, for $i=1, \ldots, r_{\rho}$. Let $f \in \mathscr{F}_{c}(K)$. Since $K$ is closed in $G$ the Tietze Extension Theorem applied to the real and imaginary parts of $f$ yields a continuous extension $f^{*}$ on $G$. Since $G$ is in [MAP], it follows from the Stone-Weierstrass Theorem that $f^{*}$ can be uniformly approximated on the compact set $K$ by representative functions associated with $\mathscr{R}(G)$. Thus, for $\varepsilon>0$, we have

$$
\left\|f^{*}-\sum_{k=1}^{N} \sum_{i, j=1}^{\operatorname{deg} \rho^{k}} c_{i j}^{k} \rho_{i j}^{k}\right\|_{K}<\varepsilon,
$$

for some $\rho^{1}, \ldots, \rho^{N}$ in $\mathscr{R}(G)$ and $c_{i j}^{k}$ in C. Since $f=\left(f^{*}\right)_{K}$, it follows from (*) applied to $\rho^{1}, \ldots, \rho^{N}$ that $f$ can be uniformly approximated to within $\varepsilon$ on $K$ by representative functions arising from $\left\{\sigma_{i}^{\rho^{k}} \mid i=1, \ldots, r_{\rho^{k}} ; k=1, \ldots, N\right\}$. Hence the representative functions associated with $\left\{\sigma_{i}^{\rho} \mid \rho \in \mathscr{R}(G), i=1, \ldots, r_{\rho}\right\}$ are uniformly dense in $\mathscr{F}_{c}(K)$. Since $K$ is compact, $\|g\|_{2} \leqq\|g\|_{K}$, for every $g$ in $\mathscr{F}_{c}(K)$, so that the representative functions arising from $\left\{\sigma_{i}^{\rho}\right\}$ are dense in $L_{2}(K)$. By the above and by the orthogonality relations for the compact group $K$ the coefficient functions of the $\sigma_{i}^{o}$ form a maximal orthonormal family in $L_{2}(K)$ and therefore the $\sigma_{i}^{o}$ comprise (up to unitary equivalence) all continuous irreducible unitary representations of $K$.

COROLlaRY 1. Let $G$ be an [MAP]-group and $K$ be a compact central subgroup. Then each group character of $K$ extends to a continuous finite-dimensional irreducible unitary representation of $G$. 
Proof. Let $\chi$ be the given group character of $K$. By Theorem 5.1, choose a continuous finite-dimensional irreducible unitary representation $\rho$ of $G$, so that $\chi$ is a component of $\rho_{K}$. As usual, $\rho(y)=\xi(y) I_{d_{\rho}}$, for $y$ in $K$, since $K$ is central and $\rho$ irreducible, where $\xi$ is some group character of $K$. But since $K$ is abelian, $\rho_{K}=\chi^{1} \oplus \cdots \oplus \chi^{r}$, where $\chi^{i}$ are 1-dimensional unitary representations of $K$, i.e., group characters, and $\chi^{1}$, say, is equivalent to $\chi$. Evidently, $\chi^{1}=\chi$. Combining the above we conclude that $\chi^{i}=\chi^{1}=\xi$, for all $i$. Hence $\chi=\xi$. Thus $\rho$ extends $\chi$.

COROLlaRY 2. Let $G$ be an [MAP]-group and $K$ be a compact subgroup of $G$. Then the restriction map $\mathscr{F}_{r}(G) \rightarrow \mathscr{F}_{r}(K)$ is surjective.

Proof. This follows directly from Theorem 5.1.

Corollary 3. Let $G$ be an [MAP]-group and $K$ a compact Lie subgroup. Then there exists a continuous finite-dimensional unitary representation $\rho$ of $G$ whose restriction to $K$ is faithful.

Proof. We first observe that any group has a faithful continuous unitary representation if and only if it has a finite number of irreducible ones which separate the points. Applying this to a faithful representation of $K$ we obtain a finite number $\sigma_{1}, \ldots, \sigma_{r}$ of irreducible representations of $K$ which separate the points. By Theorem 5.1 , choose irreducible representations $\rho_{i}$ of $G$ such that each $\rho_{i}$, when restricted to $K$, contains $\sigma_{i}$ as an irreducible component. Their direct sum satisfies the conclusion.

REMARK. Theorem 5.1 and Corollaries 2 and 3 are generalizations of facts known to hold for compact groups.

We continue this section with some results on representations of bounded degree. $G_{1}$ denotes the connected component of 1 of the group $G$.

Although the next result is well known we prefer to give a proof and then exploit the method.

Proposition 5.1. Let $G$ be a group possessing a normal abelian subgroup $A$ of finite index. Then $d_{\rho} \leqq[G: A]$ for all $\rho \in \mathscr{R}(G)$.

Proof. Let $\rho \in \mathscr{R}(G)$. Since $A$ is normal we have, by Clifford's Theorem [2],

$$
\rho_{A}=s\left(\sigma^{1} \oplus \sigma^{x_{2}} \oplus \cdots \oplus \sigma^{x_{r}}\right)
$$

where $\sigma^{x}(y)=\sigma\left(x y x^{-1}\right), x \in G, y \in A$ and the $\sigma^{x_{i}}$ are unequivalent irreducible unitary representations of $A$. Since $A$ is abelian the $\sigma^{x_{i}}$ are distinct and onedimensional. Let $\left(\sigma^{x_{i}}\right)^{G}$ be the induced representation of $\sigma^{x_{i}}$ to $G$. It is unitary and therefore semisimple. Since $A$ is of finite index it follows, by Frobenius Reciprocity [3], that $\left[\left(\sigma^{x_{i}}\right)^{G}: \rho\right]=\left[\rho_{A}: \sigma^{x_{i}}\right]=s$. Hence $\operatorname{deg}\left(\sigma^{x_{i}}\right)^{G}=\operatorname{deg} \sigma^{x_{i}}[G: A]=[G: A]$. Thus $d_{\rho} \leqq[G: A] / s \leqq[G: A]$.

The following corollary sharpens the estimate of Theorem 2.3.

COROLlaRY. Let $G$ be a discrete $[Z]$-group and $\rho \in \mathscr{R}(G)$. Then $d_{\rho} \leqq[G: Z]^{1 / 2}$. 
Proof. For any normal abelian subgroup $A$ of finite index, we have $s d_{\rho} \leqq[G: A]$. If $A=Z$ then $\rho_{Z}=\lambda I$ where $\lambda$ is one-dimensional. Thus $r=1$ and $s=d_{\rho}$, so that $d_{\rho}^{2} \leqq[G: Z]$.

REMARK. Actually, one can do better without Clifford's Theorem as follows. Suppose $[G: Z]<\infty$ and $\rho \in \mathscr{R}(G)$. Let $\chi=\lambda(\rho)$, where $\lambda$ is the map defined in the beginning of $\S 6$, and $\chi \in \hat{Z}$. Then $\chi^{G}$ is a continuous unitary representation of $G$ of degree $[G: Z]$. By reciprocity, $\left[\chi^{G}: \sigma\right]=\left[\sigma_{z}: \chi\right]=0$ if $\sigma \notin \lambda^{-1}(\chi)$ and $d_{\sigma}$ if $\sigma \in \lambda^{-1}(\chi)$. Thus $\chi^{G}=\sum_{\sigma \in \lambda^{-1}(x)} d_{\sigma} \sigma$. In particular, $[G: Z]=\sum_{\sigma \in \lambda^{-1}(x)} d_{\sigma}^{2}$ and therefore $d_{\rho}^{2} \leqq[G: Z]$.

In [10] I. Kaplansky characterized locally compact unimodular groups all of whose primitive representations are of bounded degree by polynomial identities satisfied by the group algebra. The most important applications are obtained in the case of a compact group where he proved the following theorem: If $K$ is a compact group and all the continuous irreducible unitary representations are of bounded degree then $K_{1}$ is abelian. Conversely, if $K$ is a compact Lie group and $K_{1}$ is abelian then these representations are of bounded degree. (For the definitions, see $[10]\left({ }^{12}\right)$.)

THEOREM 5.2. Let $G$ be an arbitrary locally compact group. If all the continuous irreducible unitary Hilbert space representations of $G$ are finite-dimensional and of bounded degree then $G_{1}$ is abelian.

Proof. It follows from the Gelfand-Raikov Theorem that $G$, and hence $G_{1}$, is in [MAP]. Hence, by the theorem of Freudenthal-Weil [8], $G_{1}=V \times K$ (direct product) where $V$ is a vector group and $K$ is compact. By Theorem 5.1, the continuous irreducible unitary representations of $K$ are components of the restrictions of those of $G$. Hence the continuous irreducible unitary representations of $K$ are of bounded degree. Thus $K_{1}$ is abelian, by Kaplansky's Theorem. Since $K_{1}=K, G_{1}$ is abelian.

On the other hand let $G$ be a Lie group in $[Z]$ and suppose $G_{1}$ is abelian. Then since $G_{1}$ is open in $G$ so is $Z G_{1}$. Consequently $Z G_{1}$ has finite index in $G$. Because $Z$ is central and $G_{1}$ is normal abelian, $Z G_{1}$ is normal abelian. By Proposition 5.1 all $\rho \in \mathscr{R}(G)$ are of bounded degree. Thus, by Theorem 2.1 , we have the following partial converse to Theorem 5.2.

THEOREM 5.3. Let $G$ be a Lie group in $[Z]$. If $G_{1}$ is abelian then all the continuous irreducible unitary representations of $G$ are of bounded degree.

REMARK. Both Theorems 5.2 and 5.3 generalize the corresponding results of $[10]\left({ }^{13}\right)$. In the case of a Lie group $G$, Theorem 5.3 also substantially generalizes Theorem 2.3, since there $Z$ had finite index and therefore $G_{1}$ was actually central. Further, both in Theorem 5.3 and the corresponding theorem of Kaplansky it is essential that $G$ be a Lie group, for there are many examples of compact totally

$\left.{ }^{(12}\right)$ Actually, the above results are not explicitly stated in [10] but follow directly from the theorems contained there. In part this was also pointed out by Isaacs and Passman [9].

$\left.{ }^{(13}\right)$ We have been informed by Professor C. Moore that he has also recently generalized Kaplansky's results along similar lines. 
disconnected groups with continuous irreducible representations of unbounded degree.

A direct consequence of a recent result of E. Thoma [19] is:

THEOREM 5.4. Let $G$ be a countable discrete group. Then $G$ is a $[Z]$-group if and only if (1) the continuous irreducible unitary representations of $G$ are all finite dimensional, and (2) $G$ is an FC-group $\left({ }^{14}\right)$.

Proof. For any $[Z]$-group the conjugacy classes are compact [7] and therefore finite in the case of a discrete group. Condition (1) is the conclusion of Theorem 2.1. Conversely, if $G$ is a countable discrete group then condition (1) implies that $G$ is of type $I$ in the sense of Mackey. (See, for instance, G. W. Mackey, The theory of group representations, University of Chicago Notes, 1955.) Theorem 6 of [19] tells us that $G$ has a normal abelian subgroup $A$ of finite index. Let $x_{1}, x_{2}, \ldots, x_{n}$ be a complete set of coset representatives of $G / A$. Then, by (2), $C\left(x_{i}\right)$, the centralizer of $x_{i}$, has finite index in $G$ for each $i$. Hence $\bigcap_{i=1}^{n}\left(C\left(x_{i}\right) \cap A\right)$ is a subgroup of $G$ of finite index. Since $A$ is abelian, this subgroup is central, and therefore $G \in[Z]$.

Conjecture. We believe the above to be a special case of the following more general theorem.

Let $G$ be a locally compact group. A necessary and sufficient condition for $G$ to be a $[Z]$-group is

(1) $G \in[S I N]$,

(2) $G \in[F D]^{-}$, i.e., the commutator subgroup of $G$ has compact closure.

(3) The continuous irreducible unitary representations of $G$ are all finitedimensional.

The necessity of (1) and (2) was proven in [7]. Condition (3) is the conclusion of Theorem 2.1. We remark that it is a natural generalization of the one investigated by Kaplansky.

Our next result generalizes the following classical theorem of Pontrjagin as well as our Theorem 3.1. Its proof, however, makes use of both these theorems.

THEOREM OF PONTRJAGIN. Let $G$ be a locally compact abelian group and let $H$ be $a$ closed subgroup of $G$. Then every group character $\chi$ of $H$ extends to a group character $\xi$ of $G$. Moreover, if $x_{0} \in G, x_{0} \notin H$, then $\xi$ can be chosen so that $\xi\left(x_{0}\right) \neq 0$.

TheOREM 5.5. Let $G$ be $a[Z]$-group and $H$ a closed central subgroup of $G$. Then each group character $\chi$ of $H$ extends to a continuous finite-dimensional irreducible unitary representation $\rho$ of $G$. Moreover, if $x_{0} \in G, x_{0} \notin H$, then $\rho$ can be chosen so that $\rho\left(x_{0}\right) \neq I_{d_{\rho}}$.

Proof. By Pontrjagin's Theorem, $\chi$ extends to the center $Z$ of $G$. Hence, as far as the extension of $\chi$ is concerned, we may assume that the given central subgroup is $Z$ and reserve the use of $H$ for other purposes. By the Structure Theorem [7],

$\left.{ }^{(14}\right) G$ is an $F C$-group if it has finite conjugacy classes. 
we have $G=V \times H$ (direct product), where $H$ contains a compact open normal subgroup $K$. Evidently, $V$ is central, and $K$ normal, in $G$. Let $L=Z K$. Then $L$ is an open subgroup of $G$ (since it contains $V K$ ); furthermore it is normal and it contains $Z$. Since $G \in[Z], L$ has finite index in $G$.

Consider $\chi_{K \cap Z}$. This is clearly a group character of the compact central subgroup $K \cap Z$ of $K$. Since $K$ is an [MAP]-group, it follows from Corollary 1 of Theorem 5.1 that $\chi_{K \cap Z}$ extends to a continuous finite-dimensional irreducible unitary representation $\gamma: K \rightarrow U_{n}(\mathbf{C})$. If $z_{1} k_{1}=z_{2} k_{2}$ (where $z_{i} \in Z, k_{i} \in K$ ) then $z_{2}^{-1} z_{1}=k_{2} k_{1}^{-1}$ so that $k_{2} k_{1}^{-1} \in K \cap Z$ and therefore, since $\gamma$ extends $\chi_{K \cap Z}$, we have $\gamma\left(k_{2} k_{1}^{-1}\right)$ $=\chi\left(k_{2} k_{1}^{-1}\right) I_{n}$. It follows that $\gamma\left(k_{2}\right) \gamma\left(k_{1}\right)^{-1}=\chi\left(k_{2} k_{1}^{-1}\right) I_{n}=\chi\left(z_{2}^{-1} z_{1}\right) I_{n}$ and, from this, that $\chi\left(z_{2}\right) \gamma\left(k_{2}\right)=\chi\left(z_{2}\right) \chi\left(z_{2}^{-1} z_{1}\right) \gamma\left(k_{1}\right)=\chi\left(z_{1}\right) \gamma\left(k_{1}\right)$. Now define $\sigma: L \rightarrow U_{n}(\mathbf{C})$ by $\sigma(z k)=\chi(z) \gamma(k)$. The above shows that $\sigma$ is well defined. Since $\gamma$ is a unitary representation and $|\chi(z)|=1$, for $z$ in $Z$, it follows that $\sigma$ is unitary. Moreover, by the above,

$$
\begin{aligned}
\sigma\left(k_{1} z_{1} k_{2} z_{2}\right) & =\sigma\left(z_{1} z_{2} k_{1} k_{2}\right)=\chi\left(z_{1} z_{2}\right) \gamma\left(k_{1} k_{2}\right)=\chi\left(z_{1}\right) \chi\left(z_{2}\right) \gamma\left(k_{1}\right) \gamma\left(k_{2}\right) \\
& =\left(\chi\left(z_{1}\right) \gamma\left(k_{1}\right)\right)\left(\chi\left(z_{2}\right) \gamma\left(k_{2}\right)\right)=\sigma\left(z_{1} k_{1}\right) \sigma\left(z_{2} k_{2}\right),
\end{aligned}
$$

so that $\sigma$ is a homomorphism. In addition, $\sigma_{K}=\gamma$; for if $k \in K$, then $k=1 k, 1 \in Z$, and $\sigma(k)=\chi(1) \gamma(k)=\gamma(k)$. Since $\sigma$ extends $\gamma$ and $\gamma$ is irreducible, so is $\sigma$. In order to complete the proof of the above assertion, we must show that $\sigma$ is continuous. Consider the canonical epimorphism $m: K \times Z \rightarrow K Z$ defined by $m(k, z)=k z$ and the unitary representation $\sigma^{*}$ of $K \times Z$ defined by $\sigma^{*}(k, z)=\chi(z) \gamma(k)$. Then we have the commutative diagram

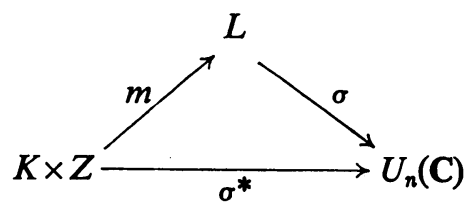

Since $\chi$ and $\gamma$ are continuous on $Z$ and $K$, respectively, it follows that $\sigma^{*}$ is continuous. Since $m$ is surjective, it follows from the above that $\sigma$ is continuous if $m$ is open; and since $L$ is open in $G$ the latter will be the case if $m: K \times Z \rightarrow G$ is an open map. Let $U$ be a neighborhood of 1 in $K \times Z$. Then $U \supseteq U_{K} \times U_{Z}$ (where $U_{K}$ and $U_{Z}$ are neighborhoods of 1 in $K$ and $Z$, respectively) and $U_{V}=V \cap U_{Z}$ is a neighborhood of 1 in $V$. Thus $m(U) \supseteq m\left(U_{K} \times U_{V}\right)=U_{K} U_{V}=U_{V} U_{K}$, and the last set is open in $G$ since $G=V \times H$ and $K$ is open in $H$. Thus $m$ is an open map and $\sigma$ is indeed continuous.

For $z$ in $Z$, we have $\sigma(z)=\sigma(z 1)=\chi(z) \gamma(1)=\chi(z) I_{n}$, by the above. Thus $\sigma$ is a continuous finite-dimensional irreducible unitary representation of $L$ which extends $\chi$ on $Z$.

Let $\rho$ be any irreducible component of the induced representation $\sigma^{G}$. Then $\rho$ is a continuous finite-dimensional irreducible unitary representation of $G$. Since 
$[G: L]$ is finite the Frobenius Reciprocity Theorem yields $\left[\rho_{L}: \sigma\right]=\left[\sigma^{G}: \rho\right]>0$. Because $L$ is normal, it follows from Clifford [2] that

$$
\rho_{L}=s\left(\sigma^{1} \oplus \sigma^{x_{2}} \oplus \cdots \oplus \sigma^{x_{r}}\right)
$$

where the notation is as in Proposition 5.1. Therefore $\left(\rho_{L}\right)_{z}=\rho_{Z}=\chi I_{s r n}$.

Now we show that there exists $\rho$ in $\mathscr{R}(G)$ so that $\rho\left(x_{0}\right) \neq I$, where $x_{0} \in G$, but $x_{0} \notin H$. If the $\rho$ found above has this property, the proof is finished. Otherwise, assume $\rho\left(x_{0}\right)=I$. Let $\pi: G \rightarrow G / H$ be the canonical epimorphism. Since $G / H$ is a [Z]-group and $x_{0} H \neq H$ there exists (by Theorem 3.1) $\sigma$ in $\mathscr{R}(G / H)$ having the property that $\sigma_{H}=I$ and $\sigma\left(x_{0} H\right) \neq I$. Evidently, $\sigma \in \mathscr{R}(G), \sigma_{H}=I$, and $\sigma\left(x_{0}\right) \neq I$. Now $(\sigma \otimes \rho)\left(x_{0}\right)=\sigma\left(x_{0}\right) \otimes \rho\left(x_{0}\right)=\sigma\left(x_{0}\right) \otimes I$ and the latter is not equivalent to $I_{d_{\rho} d_{\sigma}}$ since $\sigma\left(x_{0}\right) \neq I_{d_{\sigma}}$. On the other hand, $(\sigma \otimes \rho)_{H}=\sigma_{H} \otimes \rho_{H}=I \otimes \rho_{H}$. But $\rho_{H}=\chi I_{d_{\rho}}$. Hence $(\sigma \otimes \rho)_{H}=\chi I_{d_{\rho} d_{\sigma}}$. Now $\sigma \otimes \rho$ is a continuous, finite-dimensional, unitary representation of $G$. By semisimplicity, $\sigma \otimes \rho=\rho_{1} \otimes \cdots \otimes \rho_{r}$, where $\rho_{i} \in \mathscr{R}(G)$. Since $H$ is central, $\rho_{i}(h)=\chi_{i}(h) I_{d_{i}}$, for $h \in H$, and each $i$. Comparing this with the above result, we conclude that $\left(\chi_{i}\right)_{H}=\chi$, for each $i$, and that each $\rho_{i}$ extends $\chi$ on $H$. However, for some $i, \rho_{i}\left(x_{0}\right) \neq I_{\operatorname{deg} \rho_{i}}$; for if $\rho_{i}\left(x_{0}\right)=I_{\operatorname{deg} \rho_{i}}$, for all $i$, then $(\sigma \otimes \rho)\left(x_{0}\right)$ $=I_{d_{\rho} d_{\sigma}}$, a contradiction. Then, for that $i, \rho_{i}$ extends $\chi, \rho_{i}\left(x_{0}\right) \neq I$, and $\rho_{i} \in \mathscr{R}(G)$. (Actually, $\rho_{i}$ may not be in $\mathscr{R}(G)$; but since it is equivalent to an element of $\mathscr{R}(G)$ and since the properties in question are obviously invariant under unitary equivalence we may assume $\rho_{i} \in \mathscr{R}(G)$.)

COROLlARY. Let $G$ be $a[Z]$-group and $H$ a compactly generated central Lie subgroup. Then $G$ has a continuous finite-dimensional unitary representation $\rho$ whose restriction to $H$ is faithful.

The proof is similar to that of Corollary 3 of Theorem 5.1.

REMARK 1. Although Theorem 5.5 is a considerably better result than Corollary 1 of Theorem 5.1 (which is also used in the proof of Theorem 5.5) it does not supersede that corollary, because $[M A P]$-groups are not necessarily $[Z]$-groups. In fact, they need not even be [SIN]-groups. A pertinent counterexample is the following, adapted from S. Murakami [13]. Let $G$ be the semidirect product $H x_{\eta} K$, where $H$ is the weak direct sum of countably many copies of $Z$, discretely topologized, $K$ is the direct product of a countable number of copies of $\mathfrak{A}(Z)$, the automorphism group of $Z$, with the product topology, and $\eta\left(\left(\varepsilon_{i}\right)\right)\left(\left(x_{i}\right)\right)=\left(\varepsilon_{i}\left(x_{i}\right)\right)$. Since $G$ is totally disconnected, and nondiscrete, in order to show that $G \notin[S I N]$ it suffices to show that $G$ contains no compact normal subgroup. If $Q$ is such a subgroup let $Q^{*}=Q K$. Then $Q^{*}$ is a compact subgroup of $G$ containing $K$. We show that $Q^{*}=K$, so that $Q^{*} \subseteq K$. Since $Q^{*} \cap H=(1)$, the canonical epimorphism $G \rightarrow G / H$ is faithful on $Q^{*}$. Since $G / H \cong K$, it follows from this that $Q^{*}=K$. A direct calculation shows that $K$ contains no nontrivial normal subgroup of $G$.

For each positive integer $n$, let $H_{n}$ be the subgroup of $H$ consisting of elements of the form $\left(x_{i}\right)$ where $x_{i}=0$, for $i \leqq n ; H_{n}$ is a closed normal subgroup of $G$. Since 
$G / H$ is compact, in order to show that $G \in[M A P]$, it suffices to show that points of $H$ can be separated from the identity by continuous finite-dimensional unitary representations of $G$. Hence, since $\bigcap_{n} H_{n}=(1)$, it suffices to show that, for each $n$, $G / H_{n} \in[M A P]$. Let $K_{n}$ be the subgroup of $K$ consisting of elements of the form $\left(\varepsilon_{i}\right)$, where $\varepsilon_{i}=1$, for $i \leqq n$. It is easy to see that $H K_{n} / H_{n}$ is a normal abelian subgroup of finite index in $G / H_{n}$. Via Frobenius reciprocity it then follows that $G / H_{n} \in[M A P]$.

REMARK 2. Theorem 5.5 (and Pontrjagin's Theorem) can be slightly generalized as follows: let $G, H, \chi$ be as above, but $H$ not necessarily closed. Let $x_{0} \in G$ but $x_{0} \notin \bar{H}$, the closure of $H$ in $G$. Then there exists $\rho$ in $\mathscr{R}(G)$ which extends $\chi$ on $H$ and has the property that $\rho\left(x_{0}\right) \neq I$. Observe that since $\mathbf{T}$ is complete and $\chi$ is a continuous homomorphism $H \rightarrow \mathrm{T}, \chi$ extends (by uniform continuity) to a group character of $\bar{H}, \bar{H} \subseteq Z$. Then proceed as before, replacing $H$ by $\bar{H}$.

REMARK 3. Theorem 5.5 and Corollary 1 of Theorem 5.1 shed light on some results of $\S 3$ of [7]. There we proved that if $G \in[Z], H$ is a central subgroup of $G$, $V$ is a vector group and $\phi: H \rightarrow V$ is a continuous homomorphism then $\phi$ extends to a continuous homomorphism $\psi: G \rightarrow V$. Moreover, we showed by example that (even if $G$ were compact) the analogous statement was false if $V$ was replaced by $\mathbf{T}$ and noted that this was in marked contrast with the abelian theory. Theorem 5.5 and Corollary 1 of Theorem 5.1 show that such an extension does exist if one enlarges the representation space.

REMARK 4. The part of Theorem 5.5 asserting the existence of the extension yields a proof of Theorem 3.1 which is independent of infinite-dimensional representation theory. For let $x \in G, x \neq 1$. Since $G / Z$ is compact, in order to separate $x$ from 1 we may assume that $x \in Z$. The result then follows from the abelian theory together with the extension theorem. Thus all our results can be made to depend only on the classical theory of compact and abelian groups.

Definition. Let $G$ be a locally compact group and $\rho$ a continuous finitedimensional representation of $G$ (to $G L(n, \mathbf{C})$ ). If there is an open subgroup $H$ of $G$ on which $\rho$ is faithful we say that $\rho$ is locally faithful.

The next theorem enables us to study $[Z]$-groups with representations as above.

THEOREM 5.6. If $G$ is a Lie group in $[Z]$ then there exists a continuous finitedimensional unitary representation of $G$ which is locally faithful on $Z(G)\left({ }^{15}\right)$.

Proof. First assume that $G$ contains no vector subgroup. Now $Z(G)=V^{*} \times H^{*}$, where $V^{*}$ is a vector group and $H^{*}$ contains a compact open normal subgroup $K^{*}$. By the above assumption, $V^{*}=(1)$, so that $Z(G)$ contains a compact open normal subgroup, $K^{*}$. The latter is a compact Lie group and, as such, has a faithful continuous unitary representation $\sigma$. Since $K^{*}$ is abelian, $\sigma=\chi_{1} \oplus \cdots \oplus \chi_{r}$ where the $\chi_{i}$ are group characters of $K^{*}$. Since $\sigma$ is faithful, $\left\{\chi_{i} \mid i=1, \ldots, r\right\}$ separates the points of $K^{*}$. Since $K^{*}$ is central, there exists (by Theorem 5.5 or by Corollary 1

${ }^{(15)}$ In a later work we will show that if in addition $G$ is compactly generated, then $G$ actually has a faithful, continuous, finite-dimensional, unitary representation. 
of Theorem 5.1) $\left\{\rho_{i} \mid i=1, \ldots, r\right\}, \rho_{i} \in \mathscr{R}(G)$, where $\rho_{i}$ extends $\chi_{i}$. Let $\rho=\rho_{1} \oplus \cdots \oplus \rho_{r}$. Then $\rho$ is a continuous finite-dimensional unitary representation of $G$ which is faithful on $K^{*}$. This proves the theorem in this special case.

In general, $G=V \times H$, where $H$ contains $K$, a compact open normal subgroup. Clearly, $H$ is a Lie group. Moreover $H \in[Z]$ and $H$ contains no vector subgroup, because $H_{1}=K_{1}$. Hence, by the above, there exists a finite-dimensional continuous unitary representation $\rho$ of $H$ and a compact open subgroup $K^{*}$ of $Z(H)$ such that $\rho_{K^{*}}$ is faithful. Let $\gamma: V \rightarrow \mathrm{T}^{2 m}$ (where $m=\operatorname{dim} V$ ) be a faithful continuous unitary representation of $V$. Then $\gamma \oplus \rho$ is a continuous finite-dimensional unitary representation of $G$ which is faithful on $V \times K^{*}$. Now $Z(G)=V \times Z(H)$. Hence $V \times K^{*}$ is open in $Z(G)$.

COROLlARY. Let $G$ be a locally compact group with a center of finite index. Then $G$ is a Lie group if and only if $G$ possesses a locally faithful finite-dimensional continuous unitary representation.

Proof. If $G$ possesses such a representation then the classical theorem of Cartan states that $G$ is a Lie group. On the other hand, if $G$ is a Lie group and $[G: Z]$ is finite then Theorem 5.6 yields a continuous finite-dimensional unitary representation $\rho$ and an open subgroup $L$ of $Z(G)$ such that $\rho_{L}$ is faithful. Since $L$ is now also open in $G$ this completes the proof.

6. An orthogonality relation and a criterion for equivalence of representations. Since each continuous finite-dimensional irreducible unitary representation $\rho$ of $G$ has the property that $\rho(z)=\lambda(z) I$, where $z \in Z$ and $\lambda$ is a group character of $Z$, this gives a map $\rho \rightarrow \lambda_{\rho}$. If $\rho$ and $\sigma$ are unitarily equivalent then $\chi_{\rho}=\chi_{\sigma}$ and, in particular, $\left(\chi_{\rho}\right)_{z} d_{\rho}^{-1}=\left(\chi_{\sigma}\right)_{z} d_{\sigma}^{-1}$, i.e., $\lambda_{\rho}=\lambda_{\sigma}$. Hence we have a map $\lambda: \mathscr{R}(G) \rightarrow \mathcal{Z}$, where $\mathcal{Z}$ denotes the character group of $Z$. Theorem 5.5 asserts that if $G \in[Z]$ then $\lambda$ is surjective.

The next theorem gives an orthogonality relation analogous to one known to hold for compact groups; but it is less comprehensive than the latter.

THEOREM 6.1. Let $G \in[Z]$ and $\rho, \sigma \in \mathscr{R}(G)$, where $\rho \neq \sigma$. If $\lambda_{\rho}=\lambda_{\sigma}$, where $\lambda$ is the map defined above, then the function $x \rightarrow\left\langle\rho_{x}(u), u^{\prime}\right\rangle\left\langle\sigma_{x}(v), v^{\prime}\right\rangle^{-}$, where $u, u^{\prime} \in V_{\rho}$ and $v, v^{\prime} \in V_{\sigma}$, is in $\mathscr{F}_{c}(G / Z)$ and $\int_{G / Z}\left\langle\rho_{x}(u), u^{\prime}\right\rangle\left\langle\sigma_{x}(v), v^{\prime}\right\rangle-d \dot{x}=0$, for all $u, u^{\prime}, v, v^{\prime}$.

Proof. For $x$ in $G$ and $z$ in $Z$, we have

$$
\left\langle\rho_{x z}(u), u^{\prime}\right\rangle\left\langle\sigma_{x z}(v), v^{\prime}\right\rangle^{-}=\lambda_{\rho}(z)\left(\lambda_{\sigma}(z)\right)^{-}\left\langle\rho_{x}(u), u^{\prime}\right\rangle\left\langle\sigma_{x}(v), v^{\prime}\right\rangle^{-} .
$$

Since $\lambda_{\rho}=\lambda_{\sigma}$ and $\lambda_{\sigma}(z)^{-}=\lambda_{\sigma}(z)^{-1}$, it follows that the function in question is constant on cosets of $Z$. It is obviously continuous. Now let

$$
\phi\left(u, u^{\prime}, v, v^{\prime}\right)=\int_{G / Z}\left\langle\rho_{x}(u), u^{\prime}\right\rangle\left\langle\sigma_{x}(v), v^{\prime}\right\rangle^{-} d \dot{x} .
$$


For fixed $(u, v) \in V_{\rho} \times V_{\sigma}, \phi$ is conjugate linear in $u^{\prime}$ and linear in $v^{\prime}$. Hence there exists, for each $(u, v)$ in $V_{\rho} \times V_{\sigma}$, a linear operator $B_{u, v}: V_{\sigma} \rightarrow V_{\rho}$ so that

$$
\left\langle B_{u, v}\left(v^{\prime}\right), u^{\prime}\right\rangle=\phi\left(u, u^{\prime}, v, v^{\prime}\right),
$$

for all $u, u^{\prime}, v, v^{\prime}$. Hence, for $y$ in $G$ and $(u, v)$ in $V_{\rho} \times V_{\sigma}$,

$$
\begin{aligned}
\left\langle B_{u, v} \sigma_{y}\left(v^{\prime}\right), u^{\prime}\right\rangle=\phi\left(u, u^{\prime}, v, \sigma_{y}\left(v^{\prime}\right)\right\rangle & =\int_{G / Z}\left\langle\rho_{x}(u), u^{\prime}\right\rangle\left\langle\sigma_{x}(v), \sigma_{y}\left(v^{\prime}\right)\right\rangle^{-} d \dot{x} \\
& =\int_{G / Z}\left\langle\rho_{y x}(u), u^{\prime}\right\rangle\left\langle\sigma_{y x}(v), \sigma_{y}\left(v^{\prime}\right)\right\rangle^{-} d \dot{x}
\end{aligned}
$$

where we have used the invariance of the Haar integral. But

$$
\left\langle\rho_{y x}(u), u^{\prime}\right\rangle=\left\langle\rho_{x}(u), \rho_{y}^{-1}\left(u^{\prime}\right)\right\rangle \text { and }\left\langle\sigma_{y x}(v), \sigma_{y}\left(v^{\prime}\right)\right\rangle=\left\langle\sigma_{x}(v), v^{\prime}\right\rangle .
$$

Hence we have

$$
\left\langle B_{u, v} \sigma_{y}\left(v^{\prime}\right), u^{\prime}\right\rangle=\phi\left(u, \rho_{y}^{-1}\left(u^{\prime}\right), v, v^{\prime}\right\rangle=\left\langle B_{u, v}\left(v^{\prime}\right), \rho_{y}^{-1}\left(u^{\prime}\right)\right\rangle=\left\langle\rho_{y} B_{u, v}\left(v^{\prime}\right), u^{\prime}\right\rangle .
$$

Since this holds for all $u^{\prime}, v^{\prime}$, it follows that $B_{u, v} \sigma_{y}=\rho_{y} B_{u, v}$, for all $y \in G$ and $(u, v) \in V_{\rho} \times V_{\sigma}$. However, $\rho$ and $\sigma$ are inequivalent irreducible representations. Therefore, $B_{u, v}=0$. Hence $\phi\left(u, u^{\prime}, v, v^{\prime}\right)=0$, for all $u, u^{\prime}, v, v^{\prime}$. This completes the proof.

COROLlaRY. If $\left\{u_{1}, \ldots, u_{d_{\rho}}\right\}$ and $\left\{v_{1}, \ldots, v_{d_{\sigma}}\right\}$ are orthonormal bases in $V_{\rho}$ and $V_{\sigma}$, respectively, and $\left(\rho_{i j}(x)\right)$ and $\left(\sigma_{k l}(x)\right)$ the corresponding coordinate functions then

$$
\int_{G / Z} \rho_{i j}(x)\left(\sigma_{k l}(x)\right)^{-} d \dot{x}=0 \quad(\text { for all } i, j, k, l)
$$

and

$$
\int_{G / Z} \chi_{\rho} \bar{\chi}_{\sigma} d \dot{x}=0
$$

In order to interpret this result, let $\mathscr{F}_{\rho}$, for $\rho$ in $\mathscr{R}$, be the subspace of $L_{2}(G)$ spanned by the family $\left\{d_{\rho}^{1 / 2} w^{1 / 2} \rho_{i j} \mid i, j=1, \ldots, d_{\rho}\right\}$. These functions form an orthonormal basis for $\mathscr{F}_{\rho}$ and if $\rho, \sigma \in \mathscr{R}, \rho \neq \sigma$, and $\lambda_{\rho}=\lambda_{\sigma}$ then $\mathscr{F}_{\rho}$ and $\mathscr{F}_{\sigma}$ are orthogonal in $L_{2}(G)\left({ }^{16}\right)$. It might be conjectured that more comprehensive orthogonality relations exist than those described in Theorems 6.1 and 2.2. However, this is not the case; in fact, one has complete orthogonality if and only if the group is compact. The proof will appear elsewhere; it is related to a Plancherel formula for $[Z]$-groups. The Plancherel measure will be given explicitly.

We conclude the section by deriving criteria for the (unitary) equivalence of two continuous irreducible unitary representations of $G$.

${ }^{\left({ }^{16}\right)}$ As we will show in a subsequent publication, these spaces may be viewed as representation spaces for $G$. 
Proposition. Let $G$ be a locally compact abelian group and $\xi$ and $\eta$ group characters of $G$. Then $\xi=\eta$ if and only if $\|\xi-\eta\|_{G}<\sqrt{ } 3$.

Proof. For $x$ in $G$, we have $|\xi(x)-\eta(x)|=\left|1-\eta(x) \xi^{-1}(x)\right|$. If $\xi \neq \eta$ then $\eta \xi^{-1}$ is not identically 1 . We show that if $\chi \in \hat{G}$ (the character group of $G$ ) and $\chi$ is different from 1 then there is $x_{0} \in G$ such that $\left|\chi\left(x_{0}\right)-1\right| \geqq \sqrt{ } 3$. This implies $\|\xi-\eta\|_{G} \geqq \sqrt{ } 3$, a contradiction. Since $(\chi(G))^{-}$is a nontrivial closed subgroup of $\mathbf{T}$, we have either $(\chi(G))^{-}=\mathbf{T}$ or $(\chi(G))^{-}$, and hence $\chi(G)$, is a group of roots of unity. In the first case there exists a net $x_{v}$ in $G$ such that $\chi\left(x_{v}\right)$ converges to -1 . Then $\left|\chi\left(x_{v}\right)-1\right|$ converges to 2 .

On the other hand, if $\chi(G)$ is the group of $n$th roots of unity $(n \geqq 2)$ the worst case is clearly $n=3$, in which there exists a point $x_{0}$ such that $\left|\chi\left(x_{0}\right)-1\right|=\sqrt{ } 3$. This completes the proof.

In general this is the best possible estimate because if $\chi$ is a faithful character of $\mathbf{Z}_{3}$ then $\|\chi-1\|_{\mathbf{Z}_{3}}=\sqrt{ } 3$ and $\chi \neq 1$.

COROLLARY. If $G$ is a connected locally compact abelian group then $\xi=\eta$ if and only if $\|\xi-\eta\|_{G}<2$.

Proof. This result follows immediately from the proof of the proposition.

Again, this is the best possible result since, for any characters $\xi, \eta$,

$$
\|\xi-\eta\|_{G} \leqq\|\xi\|_{G}+\|\eta\|_{G}=2 .
$$

THEOREM 6.2. Let $G$ be $a[Z]$-group and $\rho$ and $\sigma$ be elements of $\mathscr{R}(G)$. Then $\rho=\sigma$ if and only if $\left\|\chi_{\rho}-\chi_{\sigma}\right\|_{G}<1$.

Proof. Since $\left|\chi_{\rho}(1)-\chi_{\sigma}(1)\right|<1$ it follows that $d_{\rho}=d_{\sigma}$; let $n$ denote the common value. For $z$ in $Z$, we have $\left|\chi_{\rho}(z)-\chi_{\sigma}(z)\right|=n\left|\lambda_{\rho}(z)-\lambda_{\sigma}(z)\right|$. Therefore,

$$
\left\|\lambda_{\rho}-\lambda_{\sigma}\right\|_{z}=n^{-1}\left\|\chi_{\rho}-\chi_{\sigma}\right\|_{z} \leqq n^{-1}\left\|\chi_{\rho}-\chi_{\sigma}\right\|_{G}<n^{-1} .
$$

Therefore, $\lambda_{\rho}=\lambda_{\sigma}$, by the Proposition. Hence, by Theorem 6.1, we have

$$
\int_{G} w(x) \chi_{\rho}(x)\left(\chi_{\sigma}(x)\right)^{-} d x=0=\int_{G} w(x) \chi_{\sigma}(x)\left(\chi_{\rho}(x)\right)^{-} d x
$$

in case $\rho$ and $\sigma$ are inequivalent. However, by Theorem 4.3, we have

$$
\int_{G} w(x)\left|\chi_{\rho}(x)\right|^{2} d x=1=\int_{G} w(x)\left|\chi_{\sigma}(x)\right|^{2} d x .
$$

It follows directly from the hypothesis of the theorem that

$$
w(x)\left|\chi_{\rho}(x)-\chi_{\sigma}(x)\right|^{2} \leqq w(x),
$$

for every $x$ in $G$. It follows from the above that

$$
2=\int_{G} w(x)\left|\chi_{\rho}(x)-\chi_{\sigma}(x)\right|^{2} d x \leqq \int_{G} w(x) d x .
$$


However, one sees easily from Theorem 1.2 that $\int_{G} w(x) d x=1$, so that a contradiction results.

COROLlaRY. It follows from the proof of Theorem 6.2 that if $\rho, \sigma \in \mathscr{R}(G)$ and $d_{\rho}=d_{\sigma}$ then $\rho=\sigma$ if and only if $\left\|\chi_{\rho}-\chi_{\sigma}\right\|_{G}<\sqrt{ } 2$.

\section{REFERENCES}

1. P. Cartier, Séminaire Sophus Lie, Ecole Norm. Sup. Paris, 1954/55-1955/56.

2. A. H. Clifford, Representations induced on an invariant subgroup, Ann. of Math. 38 (1937), 533-550.

3. C. Curtis and I. Reiner, Representation theory of finite groups and associative algebras, Interscience, New York, 1962.

4. R. Godement, Analyse harmonique dans les groupes centraux. I. Fonctions centrales et caractères, C. R. Acad. Sci. Paris 225 (1947), 19-21.

5. - Review of: F. I. Mautner, Infinite-dimensional representations of certain groups, Math. Rev. 12 (1951), 588.

6. - Mémoire sur la théorie des caractères dans les groupes localement compacts unimodulaires, J. Math. Pures Appl. 30 (1951), 1-110.

7. S. Grosser and M. Moskowitz, On central topological groups, Trans. Amer. Math. Soc. 127 (1967), 317-340.

8. G. Hochschild, The structure of Lie groups, Holden-Day, San Francisco, Calif., 1965.

9. I. M. Isaacs and D. S. Passman, Groups with representations of bounded degree, Canad. J. Math. 16 (1964), 299-309.

10. I. Kaplansky, Groups with representations of bounded degree, Canad. J. Math. 1 (1949), $105-112$.

11. L. Loomis, Abstract harmonic analysis, Van Nostrand, Princeton, N. J., 1953.

12. F. I. Mautner, Infinite-dimensional representations of certain groups, Proc. Amer. Math. Soc. 1 (1949), 582-584.

13. S. Murakami, Remarks on the structure of maximally almost periodic groups, Osaka Math. J. 2 (1950), 119-129.

14. L. Nachbin, On the finite-dimensionality of every irreducible unitary representation of a compact group, Proc. Amer. Math. Soc. 12 (1961), 11-12.

15. M. A. Naimark, Normed rings, Noordhoff, Groningen, 1959.

16. L. Pontrjagin, Topological groups, Princeton Univ. Press, Princeton, N. J., 1939.

17. I. Segal, The group algebra of a locally compact group, Trans. Amer. Math. Soc. 61 (1947), 69-105.

18. S. Takahashi, $A$ duality theorem for representable locally compact groups with compact commutator subgroup, Tôhoku Math. J. (2) 4 (1952), 115-121.

19. E. Thoma, Math. Ann. 153 (1964), 111-138.

20. B. L. van der Waerden, Modern algebra, Vol. 2, Ungar, New York, 1953.

21. A. Weil, L'intégration dans les groupes topologiques et ses applications, Hermann, Paris, 1953.

\section{UNIVERISTY OF Chicago, Chicago, Illinois \\ CORNELl UNIVERSITY, ITHACA, NeW YORK}

